\title{
Gorenstein-projective and semi-Gorenstein-projective modules
}

\author{
Claus Michael Ringel, Pu Zhang
}

\begin{abstract}
Let $A$ be an artin algebra. An $A$-module $M$ will be said to be semi-Gorensteinprojective provided that $\operatorname{Ext}^{i}(M, A)=0$ for all $i \geq 1$. All Gorenstein-projective modules are semiGorenstein-projective and only few and quite complicated examples of semi-Gorenstein-projective modules which are not Gorenstein-projective have been known. One of the aims of the paper is to provide conditions on $A$ such that all semi-Gorenstein-projective left modules are Gorensteinprojective (we call such an algebra left weakly Gorenstein). In particular, we show that in case there are only finitely many isomorphism classes of indecomposable left modules which are both semi-Gorenstein-projective and torsionless, then $A$ is left weakly Gorenstein. On the other hand, we exhibit a 6-dimensional algebra $\Lambda$ with a semi-Gorenstein-projective module $M$ which is not torsionless (thus not Gorenstein-projective). Actually, also the $\Lambda$-dual module $M^{*}$ is semiGorenstein-projective. In this way, we show the independence of the total reflexivity conditions of Avramov and Martsinkovsky, thus completing a partial proof by Jorgensen and Şega. Since all the syzygy-modules of $M$ and $M^{*}$ are 3-dimensional, the example can be checked (and visualized) quite easily.
\end{abstract}

Key words and phrases. Gorenstein-projective module, semi-Gorenstein-projective module, left weakly Gorenstein algebra, torsionless module, reflexive module, $t$-torsionfree module, Frobenius category, $\mho$ quiver.

2010 Mathematics Subject classification. Primary 16G10, Secondary 13D07, 16E65, 16G50, 20 G42.

Supported by NSFC 11431010

\section{Introduction.}

1.1. Notations and definitions. Let $A$ be an artin algebra. All modules will be finitely generated. Usually, the modules we are starting with will be left modules, but some constructions then yield right modules. Let $\bmod A$ be the category of all finitely generated left $A$-modules and $\operatorname{add}(A)$ the full subcategory of all projective modules.

If $M$ is a module, let $P M$ be a projective cover of $M$, and $\Omega M$ the kernel of the canonical map $P M \rightarrow M$. The modules $\Omega^{t} M$ with $t \geq 0$ are called the syzygy modules of $M$. A module $M$ is said to be $\Omega$-periodic provided that there is some $t \geq 1$ with $\Omega^{t} M=M$.

The right $A$-module $M^{*}=\operatorname{Hom}(M, A)$ is called the $A$-dual of $M$. Let $\phi_{M}: M \rightarrow M^{* *}$ be defined by $\phi_{M}(m)(f)=f(m)$ for $m \in M, f \in M^{*}$. A module $M$ is said to be torsionless provided that $M$ is a submodule of a projective module, or, equivalently, provided that $\phi_{M}$ is injective. A module $M$ is called reflexive provided that $\phi_{M}$ is bijective.

Let $\operatorname{Tr} M$ be the cokernel of $f^{*}$, where $f$ is a minimal projective presentation of $M$ (this is the canonical map $P(\Omega M) \rightarrow P M)$. Note that $\operatorname{Tr} M$ is a right $A$-module, called the transpose of $M$. 
A complete projective resolution is a (double infinite) exact sequence

$$
P^{\bullet}: \quad \cdots \longrightarrow P^{-1} \longrightarrow P^{0} \stackrel{d^{0}}{\longrightarrow} P^{1} \longrightarrow \cdots
$$

of projective left $A$-modules, such that $\operatorname{Hom}_{A}\left(P^{\bullet}, A\right)$ is again exact. A module $M$ is Gorenstein-projective, if there is a complete projective resolution $P^{\bullet}$ with $M$ isomorphic to the image of $d^{0}$.

A module $M$ will be said to be semi-Gorenstein-projective provided that $\operatorname{Ext}^{i}(M, A)=$ 0 for all $i \geq 1$. All Gorenstein-projective modules are semi-Gorenstein-projective. If $M$ is semi-Gorenstein-projective, then so is $\Omega M$. Denote by $\operatorname{gp}(A)$ the class of all Gorensteinprojective modules and by ${ }^{\perp} A$ the class of all semi-Gorenstein-projective modules. Then $\operatorname{gp}(A) \subseteq{ }^{\perp} A$. We propose to call an artin algebra $A$ left weakly Gorenstein provided that ${ }^{\perp} A=\operatorname{gp}(A)$, i.e., any semi-Gorenstein-projective module is Gorenstein-projective. (And $A$ is said to be right weakly Gorenstein if its opposite algebra $A^{\text {op }}$ is left weakly Gorenstein.)

The first aim of the paper is to provide a systematic treatment of the relationship between semi-Gorenstein-projective modules and Gorenstein-projective modules, see theorems 1.2 to 1.4. Some of these results are (at least partially) known or can be obtained from the literature, in particular see the paper [B3] by Beligiannis, but we hope that a unified, elementary and direct presentation may be appreciated.

1.2. First, we have various characterizations of the left weakly Gorenstein algebras.

Theorem. Let $A$ be an artin algebra. The following statements are equivalent:

(1) A is left weakly Gorenstein.

(2) Any semi-Gorenstein-projective module is torsionless.

(3) Any semi-Gorenstein-projective module is reflexive.

(4) For any semi-Gorenstein-projective module $M$, the map $\phi_{M}$ is surjective.

(5) For any semi-Gorenstein-projective module $M$, the module $M^{*}$ is semi-Gorenstein projective.

(6) Any semi-Gorenstein-projective module $M$ satisfies $\operatorname{Ext}^{1}\left(M^{*}, A_{A}\right)=0$.

(7) Any semi-Gorenstein-projective module $M$ satisfies $\operatorname{Ext}^{1}\left(\operatorname{Tr} M, A_{A}\right)=0$.

The equivalence of (1) and (2) was published by Huang-Huang [HH, Theorem 4.2].

1.3. The next result concerns artin algebras with finitely many indecomposable semiGorenstein-projective modules or with finitely many indecomposable torsionless modules.

Theorem. If the number of isomorphism classes of indecomposable modules which are both semi-Gorenstein-projective and torsionless is finite, then $A$ is left weakly Gorenstein and any indecomposable non-projective semi-Gorenstein-projective module is $\Omega$-periodic.

This combines two different directions of thoughts. First of all, Yoshino [Y] has shown that for certain commutative rings $R$ (in particular all artinian commutative rings) the finiteness of the number of isomorphism classes of indecomposable semi-Gorensteinprojective $R$-modules implies that $R$ is left weakly Gorenstein. This was generalized to artin algebras by Beligiannis [B3, Corollary 5.11]. Second, according to Marczinzik [M1], all torsionless-finite artin algebras (these are the artin algebras with only finitely many isomorphism classes of torsionless indecomposable modules) are left weakly Gorenstein. Note that a lot of interesting classes of artin algebras are torsionless-finite, see 3.6. 
1.4. If $\mathcal{C}$ is an extension-closed full subcategory of $\bmod A$, then the embedding of $\mathcal{C}$ into $\bmod A$ provides an exact structure on $\mathcal{C}$, called its canonical exact structure (for the basic properties of exact structures, see for example [K, Appendix A]). An exact category $\mathcal{F}$ is called a Frobenius category provided that it has enough projective and enough injective objects and that the projective objects in $\mathcal{F}$ are just the injective objects in $\mathcal{F}$. We denote by $\mathcal{P}(\mathcal{F})$ (and by $\mathcal{I}(\mathcal{F})$ ) the full subcategory of the projective (respectively injective) objects in $\mathcal{F}$.

The subcategories $\operatorname{gp}(A)$ and ${ }^{\perp} A$ are extension-closed, and with its canonical exact structure $\operatorname{gp}(A)$ is Frobenius with $\mathcal{P}(\operatorname{gp}(A))=\operatorname{add} A$ ([B2, Prop. 3.8]). Thus, if $A$ is left weakly Gorenstein, then $\mathcal{F}=\operatorname{gp}(A)$ is an extension-closed full subcategory of $\bmod A$ which is Frobenius with the canonical exact structure and satisfies $\mathcal{P}(\mathcal{F}) \subseteq{ }^{\perp} A \subseteq \mathcal{F}$. The following result shows that these properties characterize left weakly Gorenstein algebras.

Theorem. Let $A$ be an artin algebra and $\mathcal{F}$ an extension-closed full subcategory of $\bmod A$ such that $\mathcal{F}$ is a Frobenius category with respect to its canonical exact structure. If $\mathcal{P}(\mathcal{F}) \subseteq{ }^{\perp} A \subseteq \mathcal{F}$, then $A$ is left weakly Gorenstein and $\mathcal{F}=\operatorname{gp}(A)$.

A full subcategory $\mathcal{C}$ of $\bmod A$ is said to be resolving provided that it contains all the projective modules and is closed under extensions, direct summands and kernels of surjective maps. Note that ${ }^{\perp} A$ and $\operatorname{gp}(A)$ are resolving subcategories.

Corollary 1. Let $A$ be an artin algebra and $\mathcal{F}$ a resolving subcategory of $\bmod A$ with ${ }^{\perp} A \subseteq \mathcal{F}$. Assume that $\mathcal{F}$ with its canonical exact structure is a Frobenius subcategory. Then $A$ is left weakly Gorenstein and $\mathcal{F}=\operatorname{gp}(A)$.

Taking $\mathcal{F}={ }^{\perp} A$ in Theorem 1.4 we get

Corollary 2. An artin algebra $A$ is left weakly Gorenstein if and only if ${ }^{\perp} A$ with its canonical exact structure is a Frobenius subcategory.

We remark that $\operatorname{gp}(A)$ is the largest resolving Frobenius subcategory of $\bmod A$ (compare [B1, Prop. 2.13, Theorem 2.11], [B2, p.145], and [B3, p.1989]; also [ZX, Prop. 5.1]). This implies Theorem 1.4 and the two corollaries (as one of the referees has pointed out).

1.5. The $\mho$-quiver of an artin algebra $A$. The main tool used in the paper are the operator $\mho$, and the $\mho$-quiver of $A$. Here are the definitions.

Recall that a map $f: M \rightarrow M^{\prime}$ is said to be left minimal provided that any map $h: M^{\prime} \rightarrow M^{\prime}$ with $h f=f$ is an automorphism ([AR1]). A left add $(A)$-approximation will be called minimal provided that it is left minimal. We denote by $\mho M$ the cokernel of a minimal left $\operatorname{add}(A)$-approximation of $M$. (The symbol $\mho$, pronounced "agemo", should be a reminder that $\mho$ has to be considered as a kind of inverse of $\Omega$.) It turns out that the operator $\mho$ coincides with $\operatorname{Tr} \Omega \operatorname{Tr}$, which has been studied by Auslander and Reiten in [AR2]. See Subsection 4.4, and also 4.7.

Let $\omega: M \rightarrow P$ be a minimal left $\operatorname{add}(A)$-approximation with cokernel map $\pi: P \rightarrow$ $\mho M$. If $M$ is indecomposable and not projective, then the image of $\omega$ is contained in the radical of $P$, thus $\pi$ is a projective cover. If $M$ is, in addition, torsionless (so that $\omega$ is injective), then $\mho M$ is indecomposable and not projective, and $\Omega \mho M \simeq M$.

The $\mho$-quiver of $A$ has as vertices the isomorphism classes $[X]$ of the indecomposable non-projective modules $X$ and there is an arrow

$$
[X] \ldots-\cdots[\mho X]
$$


for any torsionless (indecomposable, non-projective) module $X$. (We hope that the reader is not irritated by the chosen orientation of the arrow: it corresponds to the usual convention when dealing with Ext-quivers.) A component of the $\mho$-quiver will be called an $\mho$-component; a path in the $\mho$-quiver will be called an $\mho$-path.

In the $\mho$-quiver, an arrow ending at $[X]$ starts at $[\mho X]$, thus for any vertex $[X]$, there is at most one arrow ending in $[X]$. If $[Z]$ is the start of an arrow, say $Z \simeq \mho X$ for some vertex $[X]$, then $X \simeq \Omega \mho X X \simeq \Omega Z$ implies that the arrow is uniquely determined. This shows that at any vertex of the $\mho$-quiver, at most one arrow starts and at most one arrow ends. As a consequence, we have:

Proposition. Any $\mho$-component is a linearly oriented quiver $\mathbb{A}_{n}$ with $n \geq 1$ vertices, or an oriented cycle $\widetilde{\mathbb{A}}_{n}$ with $n+1 \geq 1$ vertices, or of the form $-\mathbb{N}$, or $\mathbb{N}$, or $\mathbb{Z}$.

Note that we consider any subsets $I$ of $\mathbb{Z}$ as a quiver, with arrows from $z$ to $z-1$ (provided that both $z-1$ and $z$ belong to $I$ ). For example, the interval $\{1,2, \ldots, n\}$ is the quiver $\mathbb{A}_{n}$ with linear orientation (with 1 being the unique sink and $n$ the unique source). Here are the quivers $-\mathbb{N}$ and $\mathbb{N}$ :
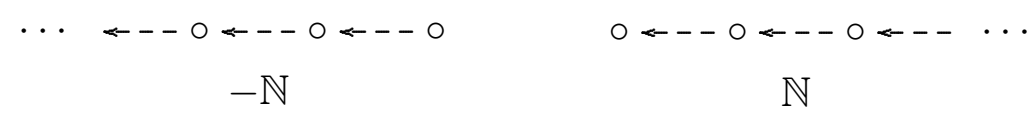

As we will see in 7.7 , all cases mentioned here can arise as $\mho$-components.

An indecomposable non-projective module $M$ will be said to be of $\mho$-type $\Delta$ where $\Delta \in\left\{\mathbb{A}_{n}, \widetilde{\mathbb{A}}_{n},-\mathbb{N}, \mathbb{N}, \mathbb{Z}\right\}$ in case the $\mho$-component containing $[M]$ is of the form $\Delta$.

Let us collect what can be read out about an indecomposable non-projective module when looking at its position in the $\mho$-quiver. Recall that a module $M$ is said to be $t$ torsionfree, provided $\operatorname{Ext}^{i}\left(\operatorname{Tr} M, A_{A}\right)=0$ for $1 \leq i \leq t$ (and $\infty$-torsionfree, provided $\operatorname{Ext}^{i}\left(\operatorname{Tr} M, A_{A}\right)=0$ for all $\left.i \geq 1\right)$; the definition is due to Auslander (see [A1, Br, AB]).

Theorem. Let $M$ be an indecomposable non-projective module.

(0) $[M]$ is an isolated vertex iff $\operatorname{Ext}^{1}(M, A) \neq 0$ and $M$ is not torsionless.

(1) $[M]$ is the start of a path of length $t \geq 1$ iff $\operatorname{Ext}^{i}(M, A)=0$ for $1 \leq i \leq t$.

In particular, $[M]$ is the start of an arrow iff $\operatorname{Ext}^{1}(M, A)=0$.

$\left(1^{\prime}\right)[M]$ is the start of an infinite path iff $M$ is semi-Gorenstein-projective.

$\left(1^{\prime \prime}\right)[M]$ is of $\mho$-type $-\mathbb{N}$ iff $M$ is semi-Gorenstein-projective, but not Gorenstein-projective.

(2) $[M]$ is the end of a path of length $t \geq 1$ iff $M$ is $t$-torsionfree for $1 \leq i \leq t$, iff $\mho^{i-1} M$ is torsionless for $1 \leq i \leq t$.

In particular, $[M]$ is the end of an arrow iff $M$ is torsionless; and $[M]$ is the end of a path of length 2 iff $M$ is reflexive.

$\left(2^{\prime}\right)[M]$ is the end of an infinite path iff $M$ is $\infty$-torsionfree, iff $M$ is reflexive and $M^{*}$ is semi-Gorenstein-projective.

$\left(2^{\prime \prime}\right)[M]$ is of $\mho$-type $\mathbb{N}$ iff $M$ is $\infty$-torsionfree, but not Gorenstein-projective.

(3) $[M]$ is the start of an infinite path and also the end of an infinite path iff $M$ is Gorenstein-projective.

$M$ is of $\mho$-type $\mathbb{Z}$ iff $M$ is Gorenstein-projective and not $\Omega$-periodic.

$M$ is of $\mho$-type $\widetilde{\mathbb{A}}_{n}$ for some $n \geq 0$ iff $M$ is Gorenstein-projective and $\Omega$-periodic. 
(4) A-duality provides a bijection between the isomorphism classes of the reflexive indecomposable $A$-modules of $\mho$-type $\mathbb{A}_{n}$ and the isomorphism classes of the reflexive indecomposable $A^{\mathrm{op}}$-modules of $\mho$-type $\mathbb{A}_{n}$.

Thus, for any $n \geq 3$, A has $\mho$-components of form $\mathbb{A}_{n}$ iff $A^{\text {op }}$ has $\mho$-components of form $\mathbb{A}_{n}$.

(5) A-duality provides a bijection between the isomorphism classes of the reflexive indecomposable $A$-modules of $\mho$-type $\mathbb{N}$ and the isomorphism classes of the reflexive indecomposable $A^{\mathrm{op}}$-modules of $\mho$-type $-\mathbb{N}$.

Thus, $A$ has $\mho$-components of form $\mathbb{N}$ iff $A^{\text {op }}$ has $\mho$-components of form $-\mathbb{N}$.

Remark 1. Characterizations of Gorenstein-projective modules. The $\mho-$ quiver shows nicely that an indecomposable module $M$ is Gorenstein-projective if and only if both $M$ and $\operatorname{Tr} M$ are semi-Gorenstein-projective, if and only if $M$ is reflexive and both $M$ and $M^{*}$ are semi-Gorenstein projective: See $\left(1^{\prime}\right),\left(2^{\prime}\right)$ and $(3)$.

Remark 2. Symmetry. The $\mho$-quiver shows a symmetry between the semi-Gorensteinprojective modules and the $\infty$-torsionfree modules: An indecomposable non-projective module $M$ is semi-Gorenstein-projective provided there is an infinite $\mho$-path starting in $M$; and $M$ is $\infty$-torsionfree, provided there is an infinite $\mho$-path ending in $M$.

Remark 3. Weakly Gorenstein algebras. An artin algebra $A$ is left weakly Gorenstein if and only if there are no modules of $\mho$-type $-\mathbb{N}$, see $\left(1^{\prime \prime}\right)$. Similarly, $A$ is right weakly Gorenstein if and only if there are no modules of $\mho$-type $\mathbb{N}$, see $\left(2^{\prime \prime}\right)$ and (5).

1.6. The first example of a semi-Gorenstein-projective module which is not Gorensteinprojective was constructed by Jorgensen and Şega [JS] in 2006, for a commutative algebra of dimension 8. Recently, Marczinzik [M2] presented some non-commutative algebras with semi-Gorenstein-projective modules which are not Gorenstein-projective. In 6.1, we exhibit a class of 6-dimensional $k$-algebras $\Lambda(q)$ with parameter $q \in k \backslash\{0\}$ and a family $M(\alpha)$ of 3-dimensional indecomposable $\Lambda(q)$-modules (with $\alpha \in k$ ) in order to find new examples:

Theorem. Let $\Lambda(q)$ be the 6-dimensional algebra defined in 6.1. If the multiplicative order of $q$ is infinite, then the $\Lambda$-modules $M(q)$ and $M(q)^{*}$ both are semi-Gorensteinprojective, but $M(q)$ is not torsionless, thus not Gorenstein-projective; all the syzygy modules $\Omega^{t} M(q)$ and $\Omega^{t}\left(M(q)^{*}\right)$ with $t \geq 0$ are 3 -dimensional and indecomposable; the module $M(q)^{* *} \simeq \Omega M(1)$ is also 3-dimensional, but decomposable.

Addendum. For any $q$, the $\Lambda(q)$-modules $M(\alpha)$ with $\alpha \in k \backslash q^{\mathbb{Z}}$ are Gorensteinprojective. Thus, if $k$ is infinite, then there are infinitely many isomorphism classes of 3-dimensional Gorenstein-projective modules.

1.7. Independence of the total reflexivity conditions. It was asked by Avramov and Martsinkowsky [AM] whether the following conditions which characterize the Gorensteinprojective modules, are independent.

(G1) The $A$-module $M$ is semi-Gorenstein-projective.

(G2) The $A$-dual $M^{*}=\operatorname{Hom}\left(M,{ }_{A} A\right)$ of $M$ is semi-Gorenstein-projective.

(G3) The $A$-module $M$ is reflexive.

Theorem. For artin algebras, the conditions (G1), (G2) and (G3) are independent. 
Proof. Theorem 1.6 provides a $\Lambda(q)$-module $M$ (namely $M=M(q)$ ) satisfying the conditions (G1), (G2) and not (G3). It remains to use the following proposition.

Proposition. If a module $M$ is semi-Gorenstein-projective and not Gorensteinprojective, then $\Omega^{2} M$ satisfies (G1) and (G3), but not (G2).

If a module $M^{\prime}$ satisfies (G1) and (G3), but not (G2), then $N=\left(M^{\prime}\right)^{*}$ is a right module satisfying (G2) and (G3), but not (G1).

Proof. Let $M$ be semi-Gorenstein-projective. Then $\Omega^{2} M$ is reflexive and semiGorenstein-projective. By Lemma 2.5, $\left(\Omega^{2} M\right)^{*}=\operatorname{Tr} M$. Thus $\left(\Omega^{2} M\right)^{*}$ is not semiGorenstein-projective (otherwise, $M$ is Gorenstein-projective).

If $M^{\prime}$ satisfies (G1) and (G3), but not (G2), then $\left(M^{\prime}\right)^{*}$ is reflexive and $\left(M^{\prime}\right)^{* *}=M^{\prime}$ is semi-Gorenstein-projective, i.e., $N=\left(M^{\prime}\right)^{*}$ satisfies (G2) and (G3), but not (G1).

Actually, for our example $A=\Lambda(q)$, there is also an $A$-module which satisfies (G2), (G3), but not (G1), namely the module $M(1)$, see 7.5 .

In [JS], where Jorgensen and Şega present the first example of a semi-Gorensteinprojective module which is not Gorenstein-projective, they also exhibited modules satisfying (G1), (G3), but not (G2), and modules satisfying (G2), (G3), but not (G1). The algebra $A$ considered in [JS] is commutative. It is an open problem whether there exists a commutative ring $A$ with a module $M$ satisfying (G1), (G2), but not (G3).

1.8. Outline of the paper. The proofs of theorems 1.2, 1.3 and 1.4 are given in sections 2, 3 and 5, respectively. We use what we call (as a shorthand) approximation sequences, namely exact sequences $0 \rightarrow X \rightarrow Y \rightarrow Z \rightarrow 0$ with $Y$ projective and $\operatorname{Ext}^{1}(Z, A)=0$, see section 2. Of special interest are the approximation sequences with both $X$ and $Z$ indecomposable and non-projective; in this case, we have $X=\Omega Z$ and $Z=\mho X$, and we call them $\mho$-sequences, see section 3 .

Section 4 deals with the $\mho$-quiver of $A$. An essential ingredient in this setting seems to be Corollary 4.4. The corresponding Remark 1 in 4.4 asserts that the kernel of the canonical map $\mho^{t} M \rightarrow\left(\mho^{t} M\right)^{* *}$ is equal to $\operatorname{Ext}^{t+1}\left(\operatorname{Tr} M, A_{A}\right)$, and its cokernel is equal to $\operatorname{Ext}^{i+2}\left(\operatorname{Tr} M, A_{A}\right)$, for all $t \geq 0$.

In sections 6 and 7 , we present the 6-dimensional algebra $\Lambda=\Lambda(q)$ depending on a parameter $q \in k \backslash\{0\}$, which we need for Theorem 1.6. We analyze some 3-dimensional representations which we denote by $M(\alpha)$ with $\alpha \in k$. Essential properties of the modules $M(\alpha)$ can be found in 6.3 to 6.5 ; they are labeled by (1) to (9). The properties (1) to (5) in 6.3 are those which are needed in order to exhibit a module, namely $M(q)$, which is semi-Gorenstein-projective, but not torsionless, provided the multiplicative order of $q$ is infinite (see 6.4). The remaining properties (6) to (9) in 6.5 show, in particular, that in case the multiplicative order of $q$ is infinite, also the $\Lambda$-dual $M(q)^{*}$ of $M(q)$ is semi-Gorensteinprojective. The proof of Theorem 1.6 and its Addendum is given in 6.7 and 6.8. In 7.1 and 7.2, some components of the $\mho$-quivers of the algebras $\Lambda$ and $\Lambda^{\mathrm{op}}$ are described.

The final sections 8 and 9 add remarks and mention some open questions.

1.9. Terminology. We end the introduction with some remarks concerning the terminology and its history. The usual reference for the introduction of Gorenstein-projective modules (under the name modules of Gorenstein dimension zero) is the Memoirs by Auslander and Bridger $[\mathrm{AB}]$ in 1969. Actually, in his thesis $[\mathrm{Br}]$, Bridger attributes the concept 
of the Gorenstein dimension to Auslander: In January 1967, Auslander gave four lectures at the Séminaire Pierre Samuel (see the notes [A1] by Mangeney, Peskine and Szpiro). In these lectures, he discussed the class of all reflexive modules $M$ such that both $M$ and $M^{*}$ are semi-Gorenstein-projective modules and denoted it by $G(A)$ ([A1, Definition 3.2.2]). Thus $G(A)$ is the class of the Gorenstein-projective modules and the conditions (G1), (G2) and (G3) served as the first definition. In [AB, Proposition 3.8], it is shown that a module $M$ belongs to $G(A)$ if and only if both $M$ and $\operatorname{Tr} M$ are semi-Gorensteinprojective. Of course, we should stress the following: Whereas some formulations in $[\mathrm{AB}]$ assume that the ring $A$ in question is a commutative noetherian ring, all the essential considerations in $[\mathrm{A} 1, \mathrm{Br}, \mathrm{AB}]$ are shown in the setting of an abelian category with enough projectives, and of the category of finitely generated modules over a, not necessarily commutative, noetherian ring. Enochs and Jenda [EJ1, EJ2] have reformulated the definition of Gorenstein-projective modules in terms of complete projective resolutions, see also [Chr]. Several other names for the Gorenstein-projective modules are in use, they are also called "totally reflexive" modules [AM], and "maximal Cohen-Macaulay" modules [Buch] and "Cohen-Macaulay" modules [B2].

We should apologize that we propose a new name for the modules $M$ with $\operatorname{Ext}^{i}(M, A)=$ 0 for all $i \geq 1$, namely semi-Gorenstein-projective. These modules have been called for example "Cohen-Macaulay modules" or "stable modules". However, the name "CohenMacaulay module" is in conflict with its established use for commutative rings, and, in our opinion, the wording "stable" may be too vague as a proper identifier. We hope that the name semi-Gorenstein-projective describes well what is going on: that there is something like a half of a complete projective resolution ("semi" means "half"). We also propose the name left weakly Gorenstein for an algebra $A$ with $\operatorname{gp}(A)={ }^{\perp} A$ (in contrast to "nearly Gorenstein" in [M2]); of course, a Gorenstein algebra $A$ satisfies $\operatorname{gp}(A)={ }^{\perp} A$, but the algebras with $\operatorname{gp}(A)={ }^{\perp} A$ seem to be quite far away from being Gorenstein. The left weakly Gorenstein algebras have also been called "algebras with condition (GC)" in [CH].

Acknowledgment. We thank Alex Martsinkovsky for providing copies of $[\mathrm{Br}]$ and [A1]. We are indebted to Lars Christensen, Henrik Holm, Zhaoyong Huang, Rene Marczinzik, Deja Wu for helpful comments. We also are grateful to two referees for carefully reading the manuscript and making valuable suggestions.

\section{Approximation sequences. Proof of Theorem 1.2.}

2.1. Lemma. Let $\epsilon: 0 \rightarrow X \stackrel{\omega}{\rightarrow} Y \stackrel{\pi}{\rightarrow} Z \rightarrow 0$ be an exact sequence with $Y$ projective. Then the following conditions are equivalent:

(i) $\omega$ is a left $\operatorname{add}(A)$-approximation.

(ii) $\operatorname{Ext}^{1}(Z, A)=0$.

(iii) The A-dual sequence $\epsilon^{*}$ of $\epsilon$ is exact.

An exact sequence $0 \rightarrow X \rightarrow Y \rightarrow Z \rightarrow 0$ with $Y$ projective satisfying the equivalent properties will be called in this paper an approximation sequence (this is just a shorthand, since it is too vague to be used in general). One may observe that the conditions (i), (ii) and (iii) are equivalent for any exact sequence $\epsilon: X \stackrel{\omega}{\rightarrow} Y \rightarrow Z \rightarrow 0$ with $Y$ projective, even if $\omega$ is not injective, but we are only interested in the short exact sequences. 
Proof of the equivalence of the properties. Since $Y$ is projective, applying $\operatorname{Hom}(-, A)$ to $\epsilon$ we get the exact sequence $0 \rightarrow Z^{*} \stackrel{\pi^{*}}{\longrightarrow} Y^{*} \stackrel{\omega^{*}}{\longrightarrow} X^{*} \rightarrow \operatorname{Ext}^{1}(Z, A) \rightarrow 0$. Note that $\omega$ is a left $\operatorname{add}(A)$-approximation if and only if $\omega^{*}$ is surjective. From this we get the equivalence of (i) and (ii) and the equivalence of (ii) and (iii).

2.2. Also the following basic lemma is well-known (see, for example $[R]$ ).

Lemma. Let $P_{-1} \stackrel{f}{\rightarrow} P_{0} \stackrel{g}{\rightarrow} P_{1}$ be an exact sequence of projective modules and let $g=$ up be a factorization with $p: P_{0} \rightarrow I$ epi and $u: I \rightarrow P_{1}$ mono. Then $P_{-1}^{*} \stackrel{f^{*}}{\longleftarrow} P_{0}^{*} \stackrel{g^{*}}{\longleftarrow} P_{1}^{*}$ is exact if and only if $u$ is a left $\operatorname{add}(A)$-approximation.

For the convenience of the reader, we insert the proof. Since $f^{*} g^{*}=(g f)^{*}=0$, we have $\operatorname{Im} g^{*} \subseteq \operatorname{Ker} f^{*}$. Assume now that $u$ is a left $\operatorname{add}(A)$-approximation and let $h \in \operatorname{Ker} f^{*}$, thus $h f=0$. Since $p$ is a cokernel of $f$, there is $h^{\prime}$ with $h=h^{\prime} p$. Since $u$ is a left $\operatorname{add}(A)$ approximation, there is $h^{\prime \prime}$ with $h^{\prime}=h^{\prime \prime} u$. Thus $h=h^{\prime} p=h^{\prime \prime} u p=h^{\prime \prime} g=g^{*}\left(h^{\prime \prime}\right)$ belongs to the image of $g^{*}$, there also $\operatorname{Ker} f^{*} \subseteq \operatorname{Im} g^{*}$.

Conversely, we assume that $\operatorname{Im} g^{*}=\operatorname{Ker} f^{*}$ and let $h: I \rightarrow A$ be a map. Then $h p f=0$, so that $f^{*}(h p)=0$. Therefore $h p$ belongs to $\operatorname{Ker} f^{*}$, thus to $\operatorname{Im} g^{*}$. There is $h^{\prime \prime} \in P_{1}^{*}$ with $h p=g^{*}\left(h^{\prime \prime}\right)=h^{\prime \prime} g=h^{\prime \prime} u p$, and therefore $h=h^{\prime \prime} u$.

This Lemma will be used in various settings, see 4.3.

2.3. A semi-Gorenstein-projective and $\Omega$-periodic module is Gorenstein-projective.

Proof. Let $M$ be semi-Gorenstein-projective and assume that $\Omega^{t} M=M$ for some $t \geq 1$. Let $\cdots \rightarrow P_{i} \rightarrow \cdots \rightarrow P_{0} \rightarrow M \rightarrow 0$ be a minimal projective resolution of $M$. Then

$$
0 \rightarrow \Omega^{t} M \rightarrow P_{t-1} \rightarrow \cdots \rightarrow P_{0} \rightarrow M \rightarrow 0
$$

is the concatenation of approximation sequences. Since $\Omega^{t} M=M$, we can concatenate countably many copies of $(+)$ in order to obtain a double infinite acyclic chain complex of projective modules. As a concatenation of approximation sequences, it is a complete projective resolution. Therefore, $M$ is Gorenstein projective.

2.4. Here are two essential observations.

(a) Let $0 \rightarrow X \rightarrow Y \rightarrow Z \rightarrow 0$ be an approximation sequence. Then $\phi_{X}$ is surjective if and only if $Z$ is torsionless. We can also say: $X$ is reflexive if and only if $Z$ is torsionless.

(b) Let $0 \rightarrow X \rightarrow Y \rightarrow Z \rightarrow 0$ be an approximation sequence. Then $\operatorname{Ext}^{1}\left(X^{*}, A_{A}\right)=0$ if and only if $\phi_{Z}$ is surjective.

Proof of (a) and (b). Since $0 \rightarrow X \stackrel{\omega}{\rightarrow} Y \stackrel{\pi}{\rightarrow} Z \rightarrow 0$ is an approximation sequence, it follows that

$$
0 \longrightarrow Z^{*} \stackrel{\pi^{*}}{\longrightarrow} Y^{*} \longrightarrow X^{*} \longrightarrow 0
$$

is an exact sequence of right $A$-modules. This induces an exact sequence

$$
0 \longrightarrow X^{* *} \longrightarrow Y^{* *} \stackrel{\pi^{* *}}{\longrightarrow} Z^{* *} \longrightarrow \operatorname{Ext}_{A}^{1}\left(X^{*}, A_{A}\right) \longrightarrow 0
$$


of left $A$-modules, and we obtain the commutative diagram

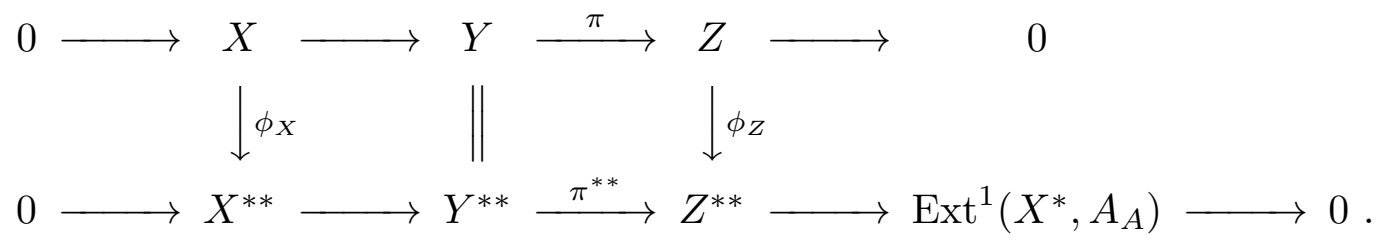

By the Snake Lemma, the kernel of $\phi_{Z}$ is isomorphic to the cokernel of $\phi_{X}$, Thus $\phi_{Z}$ is a monomorphism if and only if $\phi_{X}$ is an epimorphism. Since $X$ is torsionless, $X$ is reflexive if and only if $\phi_{X}$ is surjective. This is (a).

By the commutative diagram above, we see that $\phi_{Z}$ is epic if and only if so is $\pi^{* *}$, and if and only if $\operatorname{Ext}_{A}^{1}\left(X^{*}, A_{A}\right)=0$. This is (b).

Corollary. A module $X$ is reflexive if and only if both $X$ and $\mho X$ are torsionless.

Proof. If $X$ is reflexive, then it is torsionless. Thus we may assume from the beginning that $X$ is torsionless. Any minimal left add $(A)$-approximation $X \rightarrow Y$ is injective and its cokernel is $\mho X X$. The exact sequence $0 \rightarrow X \rightarrow Y \rightarrow \mho X \rightarrow 0$ is an approximation sequence, and 2.4 (a) asserts that $X$ is reflexive iff $\mho X$ is torsionless.

Remark. The assertion of the corollary can be strengthened as follows. For any module $X$, let us denote by $\mathrm{K} X$ the kernel of the map $\phi_{X}: X \rightarrow X^{* *}$. Of course, $\mathrm{K} X$ is the kernel of any left add $(A)$-approximation of $X$. Therefore $X$ is torsionless if and only if $\mathrm{K} X=0$. Claim: The cokernel of the map $\phi_{X}: X \rightarrow X^{* *}$ is isomorphic to $\mathrm{K} \mho X$.

Proof. Let $u: X \rightarrow Y$ be a minimal add $(A)$ approximation, say with cokernel $p: Y \rightarrow$ $\mho X$. The $A$-dual of the exact sequence $X \stackrel{u}{\rightarrow} Y \stackrel{p}{\rightarrow} \mho X \rightarrow 0$ is $0 \leftarrow X^{*} \stackrel{u^{*}}{\longleftarrow} Y^{*} \stackrel{p^{*}}{\longleftarrow}$ $(\mho X)^{*} \leftarrow 0$, since $u$ is an $\operatorname{add}(A)$-approximation. Using again $A$-duality, we obtain the exact sequence $0 \rightarrow X^{* *} \stackrel{u^{* *}}{\longrightarrow} Y^{* *} \stackrel{p^{* *}}{\longrightarrow}(\mho X)^{* *}$. Thus there is the following commutative diagram with exact rows:

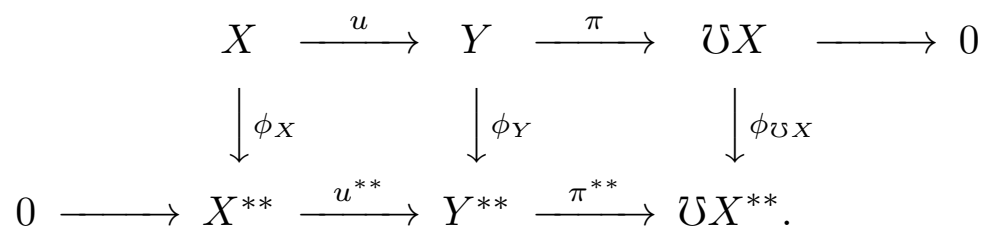

Since $\phi_{Y}$ is an isomorphism, the snake lemma yields $\operatorname{Cok} \phi_{X} \simeq \operatorname{Ker}\left(\phi_{\mho X}\right)=\mathrm{K} \mho X$.

In 4.4, we will rewrite both $\mathrm{K} X$ and $\mathrm{K} \mho X$ in order to obtain the classical AuslanderBridger sequence (see Corollary and Remark 1 in 4.4).

2.5. Lemma. Let $M$ be a module with $\operatorname{Ext}^{i}(M, A)=0$ for $i=1,2$. Then $\operatorname{Tr} M \simeq$ $\left(\Omega^{2} M\right)^{*}$ and there is a projective module $Y$ such that $M^{*} \simeq \Omega^{2} \operatorname{Tr} M \oplus Y$.

Proof: Let $\pi: P M \rightarrow M$ and $\pi^{\prime}: P \Omega M \rightarrow \Omega M$ be projective covers with inclusion maps $\omega: \Omega M \rightarrow P M$ and $\omega^{\prime}: \Omega^{2} M \rightarrow P \Omega M$. Then $\omega \pi^{\prime}$ is a minimal projective presentation of $M$. By definition, $\operatorname{Tr} M$ is the cokernel of $\left(\omega \pi^{\prime}\right)^{*}$. Since $\operatorname{Ext}^{i}(M, A)=0$ for $i=1,2$, the exact sequences

$$
0 \rightarrow \Omega^{2} M \stackrel{\omega^{\prime}}{\rightarrow} P \Omega M \stackrel{\pi^{\prime}}{\rightarrow} \Omega M \rightarrow 0, \quad 0 \rightarrow \Omega M \stackrel{\omega}{\rightarrow} P M \stackrel{\pi}{\rightarrow} M \rightarrow 0
$$


are approximation sequences. As a consequence, the corresponding $A$-dual sequences

$$
0 \leftarrow\left(\Omega^{2} M\right)^{*} \stackrel{\left(\omega^{\prime}\right)^{*}}{\longleftarrow}(P \Omega M)^{*} \stackrel{\left(\pi^{\prime}\right)^{*}}{\longleftarrow}(\Omega M)^{*} \leftarrow 0, \quad 0 \leftarrow(\Omega M)^{*} \stackrel{\omega^{*}}{\longleftarrow}(P M)^{*} \stackrel{\pi^{*}}{\longleftarrow} M^{*} \leftarrow 0
$$

are exact. The concatenation

$$
\epsilon: \quad 0 \leftarrow\left(\Omega^{2} M\right)^{*} \stackrel{\left(\omega^{\prime}\right)^{*}}{\longleftarrow}(P \Omega M)^{*} \stackrel{\left(\omega \pi^{\prime}\right)^{*}}{\longleftarrow}(P M)^{*} \stackrel{\pi^{*}}{\longleftarrow} M^{*} \leftarrow 0
$$

shows that $\left(\Omega^{2} M\right)^{*}$ is a cokernel of $\left(\omega \pi^{\prime}\right)^{*}$, thus $\operatorname{Tr} M \simeq\left(\Omega^{2} M\right)^{*}$. In addition, $\epsilon$ shows that $\Omega^{2} \operatorname{Tr} M=\Omega^{2}\left(\Omega^{2} M\right)^{*}$ is the direct sum of $M^{*}$ and a projective module $Y$.

2.6. Proof of Theorem 1.2 .

(1) implies (2) to (7): This follows directly from well-known properties of Gorensteinprojective modules. Namely, assume (1) and let $M$ be Gorenstein-projective. Then $M$ is reflexive, this yields (3), but, of course, also (2) and (4). Second, $M^{*}$ is Gorensteinprojective, thus semi-Gorenstein-projective, therefore we get (5) and (6). Finally, $\operatorname{Tr} M$ is Gorenstein-projective, thus semi-Gorenstein-projective, therefore we get (7).

Both (3) and (4) imply (2): Let $M$ be semi-Gorenstein-projective. Consider the approximation sequence $0 \rightarrow \Omega M \rightarrow P M \rightarrow M \rightarrow 0$ and note that $\Omega M$ is again semiGorenstein-projective. If (3) or just (4) holds, we know that $\phi_{\Omega M}$ is surjective, thus by 2.4 (a), $M$ is torsionless.

Both (6) and (7) imply (2): Let $M$ be semi-Gorenstein-projective. Consider the approximation sequences $0 \rightarrow \Omega M \rightarrow P M \rightarrow M \rightarrow 0$ and $0 \rightarrow \Omega^{2} M \rightarrow P M \rightarrow \Omega M \rightarrow 0$. Since $M$ is semi-Gorenstein-projective, also $\Omega^{2} M$ is semi-Gorenstein-projective. If (6) holds, we use (6) for $\Omega^{2} M$ in order to see that $\operatorname{Ext}^{1}\left(\left(\Omega^{2} M\right)^{*}, A_{A}\right)=0$. If $(7)$ holds, we use (7) for $M$ in order to see that $\operatorname{Ext}^{1}\left(\operatorname{Tr} M, A_{A}\right)=0$. According to 2.5 , we see that $\operatorname{Tr} M=\left(\Omega^{2} M\right)^{*}$. Thus in both cases (6) and (7), we have $\operatorname{Ext}^{1}\left(\left(\Omega^{2} M\right)^{*}, A_{A}\right)=0$. According to 2.4 (b), it follows from $\operatorname{Ext}^{1}\left(\left(\Omega^{2} M\right)^{*}, A_{A}\right)=0$ that $\phi_{\Omega M}$ is surjective. By 2.4 (a), $M$ is torsionless.

Trivially, (5) implies (6). Altogether we have shown that any one of the assertions (3) to (7) implies (2).

It remains to show that (2) implies (1). Let $M$ be semi-Gorenstein-projective and torsionless. We want to show that $M$ is Gorenstein-projective. Let $M_{i}=\mho^{i} M$ for all $i \geq 0$ (with $M_{0}=M$ ). Since $M_{0}$ is torsionless, there is an approximation sequence $0 \rightarrow M_{0} \rightarrow P_{1} \rightarrow M_{1} \rightarrow 0$, and $M_{1}$ is again semi-Gorenstein-projective. By assumption, $M_{1}$ is again torsionless. Inductively, starting with a torsionless module $M_{i}$, we obtain an approximation sequence $\epsilon_{i}: 0 \rightarrow M_{i} \rightarrow P_{i+1} \rightarrow M_{i+1} \rightarrow 0$, we conclude that with $M_{i}$ also $M_{i+1}$ is semi-Gorenstein-projective. By (2) we see that $M_{i+1}$ is torsionless, again. Concatenating a minimal projective resolution of $M$ with these approximation sequences $\epsilon_{i}$, for $0 \leq i$, we obtain a complete projective resolution of $M$.

\section{3. $\mho$-sequences. Proof of theorem 1.3.}

3.1. An approximation sequence $0 \rightarrow X \rightarrow Y \rightarrow Z \rightarrow 0$ will be called an $\mho$-sequence provided that both $X$ and $Z$ are indecomposable and not projective (the relevance of such sequences was stressed already in $[\mathrm{RX}]$ ). 
Lemma. An approximation sequence is the direct sum of $\mho$-sequences and an exact sequence $0 \rightarrow X^{\prime} \rightarrow Y^{\prime} \rightarrow Z^{\prime} \rightarrow 0$ with $X^{\prime}$, $Z^{\prime}$ (thus also $Y^{\prime}$ ) being projective.

Proof: Let $0 \rightarrow X \stackrel{\omega}{\rightarrow} Y \stackrel{\pi}{\rightarrow} Z \rightarrow 0$ be an approximation sequence. Since $Y$ is projective and $\pi$ is surjective, a direct decomposition $Z=Z_{1} \oplus Z_{2}$ yields a direct sum decomposition of the sequence. Since $\omega$ is a left $\operatorname{add}(A)$-approximation, there is also the corresponding assertion: If $X=X_{1} \oplus X_{2}$, then $X \stackrel{\omega}{\rightarrow} Y$ is the direct sum of two maps $X_{1} \rightarrow Y_{1}$ and $X_{2} \rightarrow Y_{2}$, thus again we obtain a direct sum decomposition of the sequence. This shows that for an indecomposable approximation sequence $0 \rightarrow X \stackrel{\omega}{\rightarrow} Y \stackrel{\pi}{\rightarrow} Z \rightarrow 0$, the modules $X$ and $Z$ are indecomposable or zero (and, of course, not both can be zero).

If $Z$ is indecomposable and projective, then the sequence $0 \rightarrow X \rightarrow Y \rightarrow Z \rightarrow 0$ splits off $0 \rightarrow 0 \rightarrow Z \stackrel{1}{\rightarrow} Z \rightarrow 0$, thus $X=0$. Similarly, if $X$ is indecomposable and projective, then the sequence $0 \rightarrow X \rightarrow Y \rightarrow Z \rightarrow 0$ splits off $0 \rightarrow X \stackrel{1}{\rightarrow} X \rightarrow 0 \rightarrow 0$, thus $Z=0$.

It remains that $0 \rightarrow X \rightarrow Y \rightarrow Z \rightarrow 0$ is an approximation sequence with both $X$ and $Z$ being indecomposable and non-projective.

3.2. Lemma. Let $\epsilon: 0 \rightarrow X \stackrel{\omega}{\rightarrow} Y \stackrel{\pi}{\rightarrow} Z \rightarrow 0$ be an exact sequence. The following conditions are equivalent:

(i) $\epsilon$ is an $\mho$-sequence.

(ii) $X$ and $Z$ are indecomposable and not projective, $\omega$ is a minimal left $\operatorname{add}(A)$-approximation, $\pi$ is a projective cover, $X=\Omega Z, Z=\mho X X$.

(iii) $X$ is indecomposable and not projective, $\omega$ is a minimal left add $(A)$-approximation.

(iv) $Z$ is indecomposable and not projective, $\pi$ is a projective cover, and $\operatorname{Ext}^{1}(Z, A)=0$.

(v) $X=\Omega Z, Y$ is projective, $Z=\mho X X$, and $X$ is indecomposable.

(vi) $X=\Omega Z, Y$ is projective, $Z=\mho X$, and $Z$ is indecomposable.

Proof: (i) implies (ii): Let $\epsilon$ be an $\mho$-sequence. Then $\omega$ has to be minimal, since otherwise $\epsilon$ would split off a non-zero sequence of the form $0 \rightarrow 0 \rightarrow P \stackrel{1}{\rightarrow} P \rightarrow 0$ with $P$ projective. Similarly, $\pi$ has to be a projective cover, since otherwise $\epsilon$ would split off a non-zero sequence of the form $0 \rightarrow P \stackrel{1}{\rightarrow} P \rightarrow 0 \rightarrow 0$. Since $\omega$ is a minimal left add $(A)$ approximation and $Z$ is the cokernel of $\omega$, we see that $Z=\mho X$. Since $\pi$ is a projective cover of $Z$ and $X$ is its kernel, $X=\Omega Z$.

(ii) collects all the relevant properties of an $\mho$-sequence. The condition (iii), (iv), (v) and (vi) single out some of these properties, thus (ii) implies these conditions.

(iii) implies (i): Since $X$ is indecomposable and not projective, $\epsilon$ has no direct summand $0 \rightarrow P \stackrel{1}{\rightarrow} P \rightarrow 0 \rightarrow 0$. Since $\omega$ is left minimal, $\epsilon$ has no direct summand $0 \rightarrow 0 \rightarrow P \stackrel{1}{\rightarrow}$ $P \rightarrow 0$. Similarly, (iv) implies (i).

Both (v) and (vi) imply (i): Since $Z=\mho X$, we have $\operatorname{Ext}^{1}(Z, A)=0$. This shows that the sequence is an approximation sequence. Since $X=\Omega Z$, the sequence $\epsilon$ has no direct summand of the form $0 \rightarrow P \stackrel{1}{\rightarrow} P \rightarrow 0 \rightarrow 0$. Since $Z=\mho X$, the sequence $\epsilon$ has no direct summand of the form $0 \rightarrow 0 \rightarrow P \stackrel{1}{\rightarrow} P \rightarrow 0$. Thus, $\epsilon$ is a direct sum of $\mho$-sequences. Finally, since $X$ or $Z$ is indecomposable, $\epsilon$ is an $\mho$-sequence.

3.3. Corollary. If $M$ is indecomposable, non-projective, semi-Gorenstein-projective, then $\Omega M$ is indecomposable, non-projective, semi-Gorenstein-projective and $M=\mho \Omega M$. 
Proof. Since $M$ is semi-Gorenstein-projective module, the canonical sequence $\epsilon: 0 \rightarrow$ $\Omega M \rightarrow P M \rightarrow M \rightarrow 0$ is an approximation sequence. Since $M$ is indecomposable and not projective, and $P M \rightarrow M$ is a projective cover, $\epsilon$ is an $\mho$-sequence, thus $\Omega M$ is indecomposable and non-projective, and $M=\mho \Omega M$, by 3.2. Of course, with $M$ also $\Omega M$ is semi-Gorenstein-projective.

3.4. Lemma. If the number of isomorphism classes of indecomposable modules which are both semi-Gorenstein-projective and torsionless is finite, then any indecomposable nonprojective semi-Gorenstein-projective module $M$ is $\Omega$-periodic.

Proof. According to 3.3, the modules $\Omega^{t} M$ with $t \geq 1$ are indecomposable modules which are torsionless and semi-Gorenstein-projective. Since there are only finitely many isomorphism classes of indecomposable torsionless semi-Gorenstein-projective modules, there are natural numbers $1 \leq s<t$ with $\Omega^{s} M=\Omega^{t} M$. Then $M=\mho^{s} \Omega^{s} M=$ $\mho^{s} \Omega^{t} M=\Omega^{t-s} M$ and $t-s \geq 1$, thus $M$ is $\Omega$-periodic.

3.5. Proof of Theorem 1.3. We assume that the number of isomorphism classes of indecomposable torsionless semi-Gorenstein-projective modules is finite. According to 3.4, any indecomposable non-projective semi-Gorenstein-projective module is $\Omega$-periodic. 2.3 shows that any semi-Gorenstein-projective $\Omega$-periodic module is Gorenstein-projective.

Remark. One of the referees has pointed out that Theorem 1.3 can be improved by replacing the class of all torsionless modules by an arbitrary full subcategory $\mathcal{X}$ which is closed under direct summands, contains add $(A)$, and contains for any indecomposable module $M$ at least one syzygy module $\Omega^{n} M$. If ${ }^{\perp} A \cap \mathcal{X}$ contains only finitely many isomorphism classes of indecomposable modules, then $\Lambda$ is left weakly Gorenstein and any Gorenstein-projective module is $\Omega$-periodic.

3.6. Torsionless-finite algebras. An artin algebra $A$ is said to be torsionless-finite if there are only finitely many isomorphism classes of indecomposable torsionless modules. Theorem 1.3 implies that any torsionless-finite artin algebra is left weakly Gorenstein, as Marczinzik [M1] has shown. If $\Lambda$ is torsionless-finite, also $\Lambda^{\text {op }}$ is torsionless-finite [R], thus a torsionless-finite artin algebras is also right weakly Gorenstein. Note that many interesting classes of algebras are known to be torsionless-finite. In particular, we have

The following algebras are torsionless-finite, hence left and right weakly Gorenstein.

(1) Algebras $A$ such that $A / \operatorname{soc}\left({ }_{A} A\right)$ is representation-finite.

(2) Algebras stably equivalent to hereditary algebras, in particular all algebras with radical square zero.

(3) Minimal representation-infinite algebras.

(4) Special biserial algebras without indecomposable projective-injective modules.

See for example $[R]$, where also other classes of torsionless-finite algebras are listed.

Chen [Che] has shown that a connected algebra $A$ with radical square zero either is self-injective, or else all the Gorenstein-projective modules are projective. The assertion that algebras with radical square zero are weakly Gorenstein complements this result. 


\section{The $\mho$-quiver.}

4.1. We recall that the $\mho$-quiver of $A$ has as vertices the isomorphism classes $[X]$ of the indecomposable non-projective modules $X$ and there is an arrow

$$
[X] \ldots-\cdots[Z]
$$

provided that $X$ is torsionless and $Z=\mho X$, thus provided that there exists an $\mho$-sequence $0 \rightarrow X \rightarrow Y \rightarrow Z \rightarrow 0$. We will also write the vertex $[X]$ simply as $X$.

\subsection{The $A$-dual of an $\mho$-sequence.}

Lemma. (a) Let $\epsilon: 0 \rightarrow X \rightarrow Y \rightarrow Z \rightarrow 0$ be an approximation sequence and assume that $X$ is reflexive. Then $\operatorname{Ext}^{1}\left(X^{*}, A_{A}\right)=0$ if and only if $Z$ is reflexive, if and only if the $A$-dual $\epsilon^{*}$ of $\epsilon$ is again an approximation sequence.

(b) Let $\epsilon: 0 \rightarrow X \rightarrow Y \rightarrow Z \rightarrow 0$ be an $\mho$-sequence with $X$ reflexive. Then $Z$ is reflexive, if and only if the $A$-dual $\epsilon^{*}$ of $\epsilon$ is again an $\mho$-sequence.

Proof. (a) By 2.4 (a), we see that $Z$ is always torsionless. Thus 2.4 (b) shows that $\operatorname{Ext}^{1}\left(X^{*}, A_{A}\right)=0$ if and only if $Z$ is reflexive. First, assume that $Z$ is reflexive. Then $\operatorname{Ext}^{1}\left(X^{*}, A_{A}\right)=0$, and therefore we see that the $A$-dual sequence $\epsilon^{*}$ is exact. We dualize a second time: the sequence $\epsilon^{* *}$ is isomorphic to the sequence $\epsilon$, since the three modules $X, Y, Z$ are reflexive. This means that $\epsilon^{* *}$ is exact, and therefore $\epsilon^{*}$ is an approximation sequence. Second, conversely, if $\epsilon^{*}$ is an approximation sequence, then it is exact, and therefore $\operatorname{Ext}^{1}\left(X^{*}, A_{A}\right)=0$, thus $Z$ is reflexive.

(b) Assume now that $\epsilon$ is an $\mho$-sequence. First, assume that $Z$ is reflexive. Since $X, Z$ both are reflexive, indecomposable and non-projective, also $X^{*}$ and $Z^{*}$ are indecomposable and non-projective, as we will show below. Thus $\epsilon^{*}$ is an $\mho$-sequence. Conversely, if $\epsilon$ is an $\mho$-sequence, then it is an approximation sequence and thus $Z$ is reflexive by (a).

We have used some basic facts about the $A$-dual $M^{*}$ of a module $M$.

(1) $M^{*}$ is always torsionless.

(2) If $M$ is non-zero and torsionless, then $M^{*}$ is non-zero.

(3) If $M$ is reflexive, indecomposable and non-projective, then $M^{*}$ is reflexive, indecomposable and non-projective.

Here are the proofs (or see for example [L, p.144]). (1) There is a surjective map $p: P \rightarrow M$ with $P$ projective. Then $p^{*}: M^{*} \rightarrow P^{*}$ is an embedding of $M^{*}$ into the projective module $P^{*}$. The assertion $(2)$ is obvious.

(3) Let $M$ be reflexive, indecomposable and non-projective. Consider a direct decomposition $M^{*}=N_{1} \oplus N_{2}$ with $N_{1} \neq 0$ and $N_{2} \neq 0$. Since $M^{*}$ is torsionless by (1), both modules $N_{1}$ and $N_{2}$ are torsionless, therefore $N_{1}^{*} \neq 0, N_{2}^{*} \neq 0$, thus there is a proper direct decomposition $M^{* *}=N_{1}^{*} \oplus N_{2}^{*}$. Since $M$ is reflexive and indecomposable, this is impossible. Thus $M^{*}$ has to be indecomposable. If $M^{*}$ is projective, then also $M^{* *}$ is projective. Again, since $M$ is reflexive, this is impossible.

It remains to show that $M^{*}$ is reflexive. Since $M^{* *}$ is isomorphic to $M$, we see that $M^{* * *}$ is isomorphic to $M^{*}$, thus the canonical map $M^{*} \rightarrow M^{* * *}$ has to be an isomorphism (since it is a monomorphism of modules of equal length). 
4.3. Lemma 2.2 outlines the importance of left add $(A)$-approximations when dealing with exact sequences of projective modules. Let us give a unified treatment of the relevance of approximation sequences and of $\mho$-sequences.

(a) An exact sequence $\cdots \longrightarrow P^{-1} \longrightarrow P^{0} \stackrel{d^{0}}{\longrightarrow} P^{1} \longrightarrow \cdots$ is a complete projective resolution if and only if it is the concatenation of approximation sequences.

$\left(\mathrm{a}^{\prime}\right)$ An indecomposable non-projective module $M$ is Gorenstein-projective if and only if $[M]$ is the start of an infinite $\mho$-path and the end of an infinite $\mho$-path.

(b) A module $M$ is semi-Gorenstein-projective if and only if a projective resolution (or, equivalently, any projective resolution) is the concatenation of approximation sequences.

$\left(\mathrm{b}^{\prime}\right)$ An indecomposable non-projective module $M$ is semi-Gorenstein-projective if and only if $[M]$ is the start of an infinite $\mho$-path.

(c) A module $M$ is reflexive and $M^{*}$ is semi-Gorenstein-projective if and only if there is an exact sequence $0 \rightarrow M \rightarrow P^{1} \rightarrow P^{2} \rightarrow \cdots$ which is the concatenation of approximation sequences.

$\left(\mathrm{c}^{\prime}\right)$ An indecomposable non-projective module $M$ is reflexive and $M^{*}$ is semi-Gorensteinprojective if and only if $[M]$ is the end of an infinite $\mho$-path.

Proof: We use that the $A$-dual of an approximation sequence is exact, thus the $A$-dual of the concatenation of approximation sequences is exact.

(a) Let $P^{\bullet}$ be a double infinite exact sequence of projective modules with maps $d^{i}: P^{i} \rightarrow P^{i+1}$ Write $d^{i}=\omega^{i} \pi^{i}$ with $\pi^{i}$ epi and $\omega^{i}$ mono. If $P^{\bullet}$ is a complete projective resolution, then the exactness of $\left(P^{\bullet}\right)^{*}$ at $\left(P^{i}\right)^{*}$ implies that $\omega^{i}$ is a left $\operatorname{add}(A)$-approximation, see 2.2. Thus $P^{\bullet}$ is the concatenation of approximation sequences.

(b) Let $\cdots \rightarrow P_{i} \rightarrow \cdots \rightarrow P_{1} \rightarrow P_{0} \rightarrow M \rightarrow 0$ be a projective resolution of $M$. write the map $P_{i+1} \rightarrow P_{i}$ as $\omega_{i} \pi_{i}$ with $\pi_{i}$ epi and $\omega_{i}$ mono. If the $A$-dual of the sequence $\cdots \rightarrow P_{i} \rightarrow \cdots \rightarrow P_{0}$ is exact, then all the maps $\omega_{i}$ with $i \geq 1$ have to be left $\operatorname{add}(A)$-approximations. This shows that the projective resolution is the concatenation of approximation sequences.

$\left(\mathrm{b}^{\prime}\right)$ Let $M$ be indecomposable, non-projective and semi-Gorenstein-projective. Since $\operatorname{Ext}^{1}(M, A)=0$, the sequence $0 \rightarrow \Omega M \rightarrow P M \rightarrow M \rightarrow 0$ is an $\mho$-sequence and $\Omega M$ is again indecomposable and non-projective. Also, $\Omega M$ is semi-Gorenstein-projective. Thus, we can iterate the procedure and obtain the infinite path

$$
\cdots \quad \leftarrow-\left[\Omega^{2} M\right] \leftarrow--[\Omega M] \leftarrow--[M]
$$

Conversely, assume that there is an infinite path starting with $[M]$, then it is of the form (*). Thus, for all $i \geq 1$, we have $\operatorname{Ext}^{i}(M, A) \simeq \operatorname{Ext}^{1}\left(\Omega^{i-1} M, A\right)=0$.

Proof of $(\mathrm{c})$ and $\left(\mathrm{c}^{\prime}\right)$. Assume that there are given approximation sequences $\epsilon_{i}: 0 \rightarrow$ $M^{i} \rightarrow P^{i+1} \rightarrow M^{i+1} \rightarrow 0$ for all $i \geq 0$, with $M^{0}=M$. Then all the modules $M^{i}$ are torsionless, thus reflexive by 2.4(a). In particular, $M$ itself is reflexive. The $A$-dual of $\epsilon_{i}$ is the sequence

$$
\epsilon_{i}^{*}: 0 \leftarrow\left(M^{i}\right)^{*} \leftarrow\left(P^{i+1}\right)^{*} \leftarrow\left(M^{i+1}\right)^{*} \leftarrow 0
$$


which again is an approximation sequence by $4.2(\mathrm{a})$. The concatenation of the sequences $\epsilon_{i}^{*}$ is a projective resolution of $M^{*}=\left(M^{0}\right)^{*}$. According to (b), $M^{*}$ is semi-Gorensteinprojective, since all the sequences $\epsilon_{i}^{*}$ are approximation sequences.

Conversely, assume that $M$ is reflexive and $M^{*}$ is semi-Gorenstein-projective. We want to construct a sequence $0 \rightarrow M \rightarrow P^{1} \rightarrow P^{2} \rightarrow \cdots$ which is the concatenation of approximation sequences. It is sufficient to consider the case where $M$ is indecomposable (in general, take the direct sum of the sequences). If $M$ is projective, then $0 \rightarrow M \rightarrow$ $M \rightarrow 0 \rightarrow \cdots$ is the concatenation of approximation sequences.

Thus, it remains to consider the case where $M$ is indecomposable and not projective. Since $M$ is torsionless, there is an $\mho$-sequence $\epsilon_{0}: 0 \rightarrow M \rightarrow P^{1} \rightarrow M^{1} \rightarrow 0$ (with $\left.M^{1}=\mho S M\right)$. Note that $M^{1}$ is indecomposable, not projective, and that the $A$-dual $\epsilon_{0}^{*}: 0 \leftarrow$ $M^{*} \leftarrow\left(P^{1}\right)^{*} \leftarrow\left(M^{1}\right)^{*} \leftarrow 0$ is exact. Since $M$ is reflexive, $M^{1}$ is torsionless by 2.4 (a). Since $M^{*}$ is semi-Gorenstein-projective, $\operatorname{Ext}^{1}\left(M^{*}, A_{A}\right)=0$, therefore $\phi_{M^{1}}$ is surjective and $\epsilon_{0}^{*}$ is an $\mho$-sequence, by 4.2. Altogether we know now that $M^{1}$ is reflexive, but also that $\left(M^{1}\right)^{*}=\Omega\left(M^{*}\right)$. With $M^{*}$ also $\Omega\left(M^{*}\right)$ is semi-Gorenstein-projective.

Thus $M^{1}$ satisfies again the assumptions of being indecomposable, not projective, reflexive and that its $A$-dual $\left(M^{1}\right)^{*}$ is semi-Gorenstein-projective. Thus we can iterate the procedure for getting the next $\mho$-sequence $\epsilon_{1}: 0 \rightarrow M^{1} \rightarrow P^{2} \rightarrow M^{2} \rightarrow 0$, with $M^{2}=\mho^{2} M$, and so on. Altogether, we obtain the infinite path:

$$
[M] \cdots-\cdots[\mho \mho M] \leftarrow-\left[\mho^{2} M\right] \ldots-\cdots
$$

This completes the proof of $\left(\mathrm{c}^{\prime}\right)$ and thus also of $(\mathrm{c})$.

$\left(\mathrm{a}^{\prime}\right)$ This follows immediately from $\left(\mathrm{b}^{\prime}\right)$ and $\left(\mathrm{c}^{\prime}\right)$.

4.4. For any module $M$, we have denoted by $\mathrm{K} M$ the kernel of $\phi_{M}: M \rightarrow M^{* *}$. We are going to identify $\mathrm{K} M$ with $\operatorname{Ext}^{1}\left(\operatorname{Tr} M, A_{A}\right)$. Compare [A2, Proposition 6.3]. As a consequence, we see that $\mho M=\operatorname{Tr} \Omega \operatorname{Tr} M$.

Lemma. Let $M$ be a module. Then $\operatorname{Ext}^{1}\left(\operatorname{Tr} M, A_{A}\right) \simeq \mathrm{K} M$ and there is a right module $Q$ such that $\Omega \operatorname{Tr} M \simeq \operatorname{Tr} \mho M \oplus Q$. As a consequence, $\mho M \simeq \operatorname{Tr} \Omega \operatorname{Tr} M$, thus $\mho^{t}(M) \cong \operatorname{Tr} \Omega^{t} \operatorname{Tr}(M)$ for $t \geq 1$.

Proof. Let $P^{0} \stackrel{f}{\rightarrow} P^{1} \stackrel{p}{\rightarrow} M \rightarrow 0$ be a minimal projective presentation of $M$. Thus $\operatorname{Tr} M$ is the cokernel of $f^{*}$. Let $g^{\prime}: M \rightarrow P^{2}$ be a minimal left add $(A)$-approximation. Then $\mathrm{K} M$ is the kernel of $g^{\prime}$, thus $g^{\prime}=u q$, where $q: M \rightarrow M / \mathrm{K} M$ is the canonical projection and $u$ is injective. Let $g=g^{\prime} p=u q p$. The composition

$$
P^{0} \stackrel{f}{\rightarrow} P^{1} \stackrel{g}{\rightarrow} P^{2}
$$

is zero and the homology $H\left(P^{0} \stackrel{f}{\rightarrow} P^{1} \stackrel{g}{\rightarrow} P^{2}\right)$ is just $\mathrm{K} M$, since $\operatorname{Ker}(g) / \operatorname{Im}(f) \simeq$ $\operatorname{Ker}(q p) / \operatorname{Ker}(p) \simeq \mathrm{K} M$.

We claim that the $A$-dual sequence

$$
\left(P^{0}\right)^{*} \stackrel{f^{*}}{\longleftarrow}\left(P^{1}\right)^{*} \stackrel{g^{*}}{\longleftarrow}\left(P^{2}\right)^{*}
$$


is exact. Since $g f=0$, we have $f^{*} g^{*}=0$. Conversely, let $h: P^{1} \rightarrow A$ be in the kernel of $f^{*}$, thus $h f=0$. Therefore $h$ factors through $p=\operatorname{Cok} f$, say $h=h^{\prime} p$ with $h^{\prime}: M \rightarrow A$. Since $u q$ is a left $\operatorname{add}(A)$-approximation, we obtain $h^{\prime \prime}: P^{2} \rightarrow A$ with $h^{\prime}=h^{\prime \prime} u q$. Thus $h=h^{\prime} p=h^{\prime \prime} u q p=h^{\prime \prime} g=g^{*}\left(h^{\prime \prime}\right)$ is in the image of $g^{*}$.

Since the cokernel of $f^{*}$ is $\operatorname{Tr} M$, it follows that $(*)$ is the begin of a projective resolution of $\operatorname{Tr} M$ and hence $\operatorname{Ext}^{1}\left(\operatorname{Tr} M, A_{A}\right)$ is obtained by applying $\operatorname{Hom}(-, A)$ to $(*)$ and taking the homology at the position 1 . Applying $\operatorname{Hom}(-, A)$ to $(*)$ we retrieve the sequence $P^{0} \stackrel{f}{\rightarrow} P^{1} \stackrel{g}{\rightarrow} P^{2}$, thus $\operatorname{Ext}^{1}\left(\operatorname{Tr} M, A_{A}\right)$ is equal to $H\left(P^{0} \stackrel{f}{\rightarrow} P^{1} \stackrel{g}{\rightarrow} P^{2}\right) \simeq \mathrm{K} M$. This is the first assertion.

By definition, the cokernel of $g^{\prime}$ is $\mho M$. Thus the cokernel of $g$ is $\mho M$, and therefore Cok $g^{*} \simeq \operatorname{Tr} \mho M \oplus Q^{\prime}$ for some projective right module $Q^{\prime}$. Now $\operatorname{Cok} g^{*}=\operatorname{Im} f^{*}$, since $(*)$ is exact. Since $\operatorname{Cok} f^{*}=\operatorname{Tr} M$, we have $\Omega \operatorname{Tr} M \simeq \operatorname{Im} f^{*} \oplus Q^{\prime \prime}$ for some projective right module $Q^{\prime \prime}$. This shows that $\Omega \operatorname{Tr} M \simeq \operatorname{Im} f^{*} \oplus Q^{\prime \prime}=\operatorname{Cok} g^{*} \oplus Q^{\prime \prime} \simeq \operatorname{Tr} \mho M \oplus Q^{\prime} \oplus Q^{\prime \prime}=$ $\operatorname{Tr} \mho M \oplus Q$ with $Q=Q^{\prime} \oplus Q^{\prime \prime}$. This is the second assertion.

Applying $\operatorname{Tr}$ to the isomorphism $\Omega \operatorname{Tr} M \simeq \operatorname{Tr} \mho M \oplus Q$, one obtains $\operatorname{Tr} \Omega \operatorname{Tr} M \simeq$ $\operatorname{Tr}(\operatorname{Tr} \mho M \oplus Q)=\operatorname{Tr} \operatorname{Tr} \mho M$. Since $\mho M$ has no non-zero projective direct summand, one gets $\operatorname{Tr} \operatorname{Tr} \mho M \simeq \mho M$. Thus $\mho M \simeq \operatorname{Tr} \operatorname{Tr} \mho M \simeq \operatorname{Tr} \mho \operatorname{Tr} M$.

Corollary. Let $M$ be a module. Then for all $t \geq 0$ one has

$$
\mathrm{Ext}^{t+1}\left(\operatorname{Tr} M, A_{A}\right) \simeq \mathrm{K}\left(\mho^{t} M\right)
$$

In particular, $\mho^{t} M$ is torsionless if and only if $\operatorname{Ext}^{t+1}\left(\operatorname{Tr} M, A_{A}\right)=0$. Also, $\Omega^{t} \operatorname{Tr} M \simeq$ $\operatorname{Tr} \mho^{t} M \oplus Q_{t}$ for some projective right module $Q_{t}$.

Proof. By induction on $t$, one has $\Omega^{t} \operatorname{Tr} M \simeq \operatorname{Tr} \mho^{t} M \oplus Q_{t}$ for some projective right module $Q_{t}$. It implies that $\operatorname{Ext}^{t+1}\left(\operatorname{Tr} M, A_{A}\right) \simeq \operatorname{Ext}^{1}\left(\Omega^{t} \operatorname{Tr} M, A_{A}\right) \simeq \operatorname{Ext}^{1}\left(\operatorname{Tr} \mho^{t} M, A_{A}\right)$ and thus $\operatorname{Ext}^{1}\left(\operatorname{Tr} \mho^{t} M, A_{A}\right) \simeq K\left(\mho^{t} M\right)$.

Remark 1. For any $t \geq 0$, there is an exact sequence of the form

$$
0 \rightarrow \operatorname{Ext}^{t+1}\left(\operatorname{Tr} M, A_{A}\right) \rightarrow \mho^{t} M \stackrel{\phi_{\mho^{t} M}}{\longrightarrow}\left(\mho^{t} M\right)^{* *} \rightarrow \operatorname{Ext}^{t+2}\left(\operatorname{Tr} M, A_{A}\right) \rightarrow 0 .
$$

If $t=0$, it is the classical Auslander-Bridger sequence $0 \rightarrow \operatorname{Ext}^{1}\left(\operatorname{Tr} M, A_{A}\right) \rightarrow M \rightarrow$ $M^{* *} \rightarrow \operatorname{Ext}^{2}\left(\operatorname{Tr} M, A_{A}\right) \rightarrow 0$ (see [AB], also [ARS]).

Proof. The corollary asserts that the kernel of the map $\phi_{\mho^{t} M}: \mho^{t} M \rightarrow\left(\mho^{t} M\right)^{* *}$ is isomorphic to $\mathrm{Ext}^{t+1}\left(\operatorname{Tr} M, A_{A}\right)$. On the other hand, the Remark at the end of 2.4 shows that $\operatorname{Cok} \phi_{\mho^{t} M} \simeq \mathrm{K} \mho^{t+1} M$. Since $\mathrm{K} \mho^{t+1} M \simeq \operatorname{Ext}^{1}\left(\operatorname{Tr} \mho^{t+1} M, A_{A}\right) \simeq \operatorname{Ext}^{1}\left(\Omega^{t+1} \operatorname{Tr} M, A_{A}\right) \simeq$ $\operatorname{Ext}^{t+2}\left(\operatorname{Tr} M, A_{A}\right)$, it follows that $\operatorname{Cok} \phi_{\mho^{t} M} \simeq \operatorname{Ext}^{t+2}\left(\operatorname{Tr} M, A_{A}\right)$.

Remark 2. If $M$ is any module, $\mho \operatorname{Tr} M \simeq \operatorname{Tr} \Omega M$.

Proof: There is a projective module $P$ such that $\operatorname{Tr} \operatorname{Tr} M \oplus P \simeq M$. According to Lemma 4.4 we have $\mho \operatorname{Tr} M \simeq \operatorname{Tr} \Omega \operatorname{Tr} \operatorname{Tr} M=\operatorname{Tr} \Omega(\operatorname{Tr} \operatorname{Tr} M \oplus P) \simeq \operatorname{Tr} \Omega M$.

Remark 3. In contrast to the isomorphism given in Remark 2, the right modules $\Omega \operatorname{Tr} M$ and $\operatorname{Tr} \mho M$ discussed in the lemma do not have to be isomorphic. For example, 
let $M$ be a module with $M^{*}=0$. Then $\mho M=0$, thus $\operatorname{Tr} \mho M=0$. On the other hand, if $f: P_{1} \rightarrow P(M)$ is a minimal projective presentation of $M$, then the kernel of $f^{*}$ is $M^{*}$, thus zero, and therefore $\Omega \operatorname{Tr} M \simeq(P(M))^{*}$. Thus, we see that the right module $Q$ with $\Omega \operatorname{Tr} M \simeq \operatorname{Tr} \mho M \oplus Q$ may be non-zero.

\subsection{Modules at the end of an $\mho$-path of length $t$.}

Proposition. Let $M$ be any module and $t \geq 1$. The following conditions are equivalent: (i) $\mho^{i-1} M$ is torsionless for $1 \leq 1 \leq t$.

(ii) $M$ is t-torsionfree (thus $\operatorname{Ext}^{i}\left(\operatorname{Tr} M, A_{A}\right)=0$ for $\left.1 \leq i \leq t\right)$.

If $M$ is indecomposable and not projective, then these conditions are equivalent to

(iii) $M$ is the end of an $\mho$-path of length $t$.

Already the special cases $t=1$ and $t=2$ are of interest (but well-known): $A$ module $M$ is 1-torsionfree iff $M$ is torsionless (this is case $t=1$ ); a module $M$ is 2-torsionfree iff both $M$ and $\Omega M$ are torsionless, thus iff $M$ is reflexive (this is the case $t=2$, taking into account Corollary 2.4). These special cases $t=1$ and $t=2$ are discussed at several places; let us refer in particular to [ARS], Corollary IV.3.3. Our general proof is inspired by [AB].

Proof of Proposition. For the equivalence of (i) and (ii), see Corollary in 4.4: It asserts for any $i \geq 1$, that $\mho^{i-1} M$ is torsionless iff $\operatorname{Ext}^{i}\left(\operatorname{Tr} M, A_{A}\right)=0$.

In order to show the equivalence of (i) and (iii), let $M$ be indecomposable and not projective. If (iii) is satisfied, there is an $\mho$-path of length $t$ ending in $M$. This path has to be $\mho^{t} M, \mho^{t-1} M, \ldots, \mho M, M$. This shows that for any module $\mho^{i} M$ with $0 \leq i<t$, there is an arrow starting in $\mho^{i} M$, and therefore $\mho^{i} M$ has to be torsionless.

Conversely, assume that (i) is satisfied. We show (iii) by induction on $t$. For any $t \geq 1$, there is the arrow $\mho M \rightarrow M$, since $M$ is indecomposable, non-projective and torsionless. According to 3.2 , the module $\mho M$ is again indecomposable and non-projective. Thus, if $t \geq 2$, we can use induction in order to obtain a path of length $t-1$ ending in $\mho S M$, since all the modules $\mho^{i}(\mho M)$ with $0 \leq i<t-1$ are torsionless.

\subsection{Proof of Theorem 1.5.}

(1) follows from the fact that $\operatorname{Ext}^{t}(M, A)=\operatorname{Ext}^{t-1}(\Omega M, A)$ for $t \geq 2$. For the special case $t=2$, see Corollary 2.4. (2) is Proposition 4.5. For $\left(1^{\prime}\right),\left(2^{\prime}\right)$ and $(3)$, see 4.3. For (4) and (5), we refer to $4.2(\mathrm{~b})$. Note that in an $\mho$-component of the form $\mathbb{A}_{n}$ with $n \geq 3$, as well as in those of the form $-\mathbb{N}$, all but precisely two vertices are the isomorphism classes of reflexive modules, whereas any vertex of an $\mho$-component of the form $\mathbb{N}$ is the isomorphism class of a reflexive module.

4.7. The adjoint functors $\mho$ and $\Omega$. Here we collect some important properties of the construction $\mho$. Some details of the proofs are left to the reader, since the assertions are not needed in the paper.

If $\mathcal{C}^{\prime} \subseteq \mathcal{C}$ are full subcategories of $\bmod A$, let $\mathcal{C} / \mathcal{C}^{\prime}$ be the category with the same objects as $\mathcal{C}$ such that $\operatorname{Hom}_{\mathcal{C} / \mathcal{C}^{\prime}}(X, Y)$ is the factor group of $\operatorname{Hom}_{\mathcal{C}}(X, Y)$ modulo the subspace of all maps $X \rightarrow Y$ which factor through a direct sum of modules in $\mathcal{C}^{\prime}$.

(1) The functor $\mho$ is the left adjoint of the endo-functor $\Omega$ of $\bmod A / \operatorname{add} A$. Direct verification is easy. But we should also add that Auslander and Reiten have shown in [AR2, Corollary 3.4] that the functor $\operatorname{Tr} \Omega \operatorname{Tr}$ is left adjoint to $\Omega$, and we have identified in 4.4 the functors $\mho$ and $\operatorname{Tr} \Omega \operatorname{Tr}$. 
(2) Let $\mathcal{L}(A)$ be the full subcategory of all torsionless modules, and $\mathcal{Z}(A)$ the full subcategory of all modules $Z$ with $\operatorname{Ext}^{1}(Z, A)=0$. For any module $M$, the module $\Omega M$ belongs to $\mathcal{L}(A)$, and the module $\mho M$ belongs to $\mathcal{Z}(A)$; in addition, $\mho M$ has no non-zero projective direct summand.

(3) If $Z$ satisfies $\operatorname{Ext}^{1}(Z, A)=0$ and has no non-zero projective direct summand, then $\mho \Omega Z \simeq Z$ (see 3.2). If $X$ is torsionless and has no non-zero projective direct summand, then $\Omega \mho X \simeq X$ (see 1.5 or also 3.2). In this way, one shows that the functors $\Omega$ and $\mho$ provide inverse categorical equivalences

$$
\mathcal{L}(A) / \operatorname{add}(A) \underset{\Omega}{\stackrel{\mho}{\rightleftarrows}} \mathcal{Z}(A) / \operatorname{add}(A)
$$

(4) Thus, $\Omega$ and $\mho$ provide inverse bijections between isomorphism classes as follows:

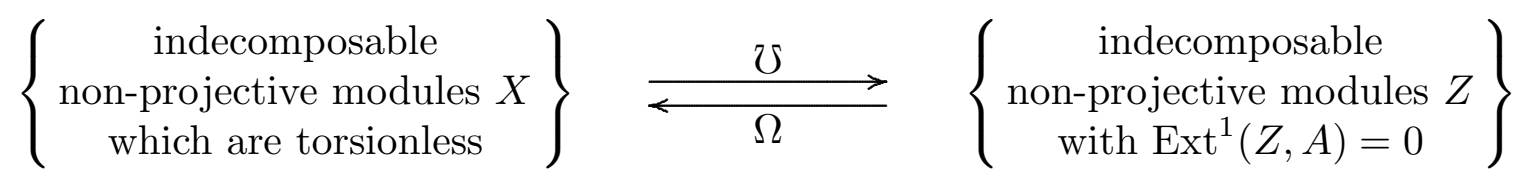

The arrows of the $\mho$-quiver visualize this bijection.

4.8. Gorenstein algebras. Recall that an artin algebra $A$ is said to be $d$-Gorenstein provided that the injective dimension of both ${ }_{A} A$ and $A_{A}$ is equal to $d$. Of course, any algebra of global dimension $d$ is $d$-Gorenstein The following result of Beligiannis [B2, Proposition 4.4] yields additional examples of weakly Gorenstein algebras.

Proposition. Let $A$ be an artin algebra and assume that the injective dimension of ${ }_{A} A$ is at most $d$. Then $A$ is right weakly Gorenstein and any module of the form $\Omega^{d} M$ is semi-Gorenstein projective.

Proof: Since the injective dimension of ${ }_{A} A$ is at most $d$, one knows that for any module $M$, the syzygy module $\Omega^{d} M$ is semi-Gorenstein-projective. [Namely, for all $i \geq 1$, we have $\operatorname{Ext}^{i}\left(\Omega^{d} M, A\right)=\operatorname{Ext}^{d+i}(M, A)=\operatorname{Ext}^{i}\left(M, \Sigma^{d} A\right)=0$; here, $\Sigma N$ denotes the cokernel of an injective envelope of a module $N$.] This implies that $A$ cannot have any indecomposable module of $\mho$-type $\mathbb{N}$. [Namely, if $M$ is of $\mho$-type $\mathbb{N}$, then $M$ is $\infty$-torsionfree and therefore $M=\Omega^{d}\left(\mho^{d} M\right)$. But as we have seen, this implies that $M$ is semi-Gorenstein-projective, therefore Gorenstein-projective. Thus $M$ is of $\mho$-type $\mathbb{Z}$ and not $\mathbb{N}$.] Therefore $A$ is right weakly Gorenstein.

Corollary 1. Let $A$ be d-Gorenstein. If an indecomposable non-projective module $M$ belongs to an $\mho$-path of length $d$, then $M$ is Gorenstein-projective. If the global dimension of $A$ is $d$, then there is no $\mho$-path of length $d$.

Proof. Since the inj. dim. ${ }_{A} A=d, A$ is right weakly Gorenstein and any module $\Omega^{d} M$ is semi-Gorenstein-projective. Since inj. $\operatorname{dim} . A_{A}$ is finite, $A$ is also left weakly Gorenstein, thus the modules $\Omega^{d} M$ are even Gorenstein-projective.

Corollary 2. If $A$ is d-Gorenstein, then $A$ has no $\mho$-component of form $-\mathbb{N}, \mathbb{N}$ or $\mathbb{A}_{n}$ with $n>d$. If the global dimension of $A$ is $d$, then any $\mho$-component is of form $\mathbb{A}_{n}$ with $n \leq d$. 


\section{Proof of Theorem 1.4.}

Since $\operatorname{add}(A) \subseteq{ }^{\perp} A \subseteq \mathcal{F}$, we see that $\operatorname{add}(A) \subseteq \mathcal{P}(\mathcal{F})=\mathcal{I}(\mathcal{F})$. $\operatorname{Thus}^{\operatorname{Ext}_{A}}{ }_{A}^{1}(X, A)=0$, for all $X \in \mathcal{F}$.

For $X \in \mathcal{F}$, there is an exact sequence $0 \longrightarrow K \longrightarrow Q \longrightarrow X \longrightarrow 0$ with $Q \in \mathcal{P}(\mathcal{F})$ and $K \in \mathcal{F}$. By $\mathcal{P}(\mathcal{F}) \subseteq{ }^{\perp} A$ we have $Q \in{ }^{\perp} A$. Thus $\operatorname{Ext}_{A}^{1}(X, A)=0$ and $\operatorname{Ext}_{A}^{m+1}(X, A)=$ $\operatorname{Ext}_{A}^{m}(K, A)$ for $m \geq 1$. So $\operatorname{Ext}_{A}^{2}(X, A)=0$, and in particular $\operatorname{Ext}_{A}^{2}(K, A)=0$. Repeating this process we see that $X \in{ }^{\perp} A$. Thus $\mathcal{F} \subseteq{ }^{\perp} A$, and hence ${ }^{\perp} A=\mathcal{F}$ is Frobenius.

For $L \in \mathcal{P}\left({ }^{\perp} A\right)$, consider an exact sequence $0 \longrightarrow K \longrightarrow P \longrightarrow L \longrightarrow 0$ with $P \in \operatorname{add}(A)$. Since $L$ and $P$ are in ${ }^{\perp} A, K \in{ }^{\perp} A$. So $\operatorname{Ext}_{A}^{1}(L, K)=0$, thus the exact sequence splits and $L \in \operatorname{add}(A)$. This shows $\mathcal{P}\left({ }^{\perp} A\right) \subseteq \operatorname{add}(A) \subseteq \mathcal{P}\left({ }^{\perp} A\right)$, and hence $\mathcal{P}\left({ }^{\perp} A\right)=\operatorname{add}(A)$.

Now consider $X \in^{\perp} A$. Since ${ }^{\perp} A$ is Frobenius, there is an exact sequence $0 \longrightarrow X \longrightarrow$ $I \longrightarrow C \longrightarrow 0$ with $I \in \mathcal{I}\left({ }^{\perp} A\right)=\mathcal{P}\left({ }^{\perp} A\right)=\operatorname{add}(A)$ and $C \in{ }^{\perp} A$. So $X$ is torsionless. This shows that $A$ is left weakly Gorenstein, according to Theorem 1.2.

\section{An example.}

Let $k$ be a field and $q \in k \backslash\{0\}$. We consider a 6-dimensional local algebra $\Lambda=\Lambda(q)$. If $k$ is infinite, then we show that there are infinitely many Gorenstein-projective $\Lambda$-modules of dimension 3. Let $o(q)=\left|q^{\mathbb{Z}}\right|$ be the multiplicative order of $q$. If $o(q)$ is infinite, we show that there is also a semi-Gorenstein-projective $\Lambda$-module of dimension 3 which is not Gorenstein-projective.

6.1. The algebra $\Lambda=\Lambda(q)$. The algebra $\Lambda$ is generated by $x, y, z$, subject to the relations:

$$
x^{2}, y^{2}, z^{2}, y z, x y+q y x, x z-z x, z y-z x .
$$

The algebra $\Lambda$ has a basis $1, x, y, z, y x$, and $z x$ and may be visualized as follows:

$\Lambda$ :

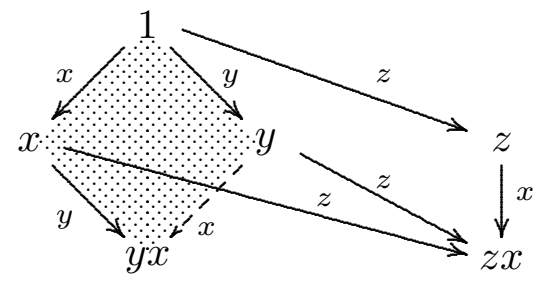

Here, we use the following convention: The vertices are the elements of the basis, the arrows are labeled by $x, y, z$. A solid arrow $v \rightarrow v^{\prime}$ labeled say by $x$ means that $x v=v^{\prime}$, a dashed arrow $v \rightarrow v^{\prime}$ labeled by $x$ means that $x v$ is a non-zero multiple of $v^{\prime}$ (in our case, $x y=-q y x)$. If $v$ is a vertex and no arrow starting at $v$ is labeled say by $x$, then $x v=0$.

One diamond in the picture has been dotted in order to draw attention to the relation $x y+q y x$; this relation plays a decisive role when looking at $\Omega M$ for a given $\Lambda$-module $M$.

We study the following modules $M(\alpha)$ with $\alpha \in k$. The module $M(\alpha)$ has a basis $v, v^{\prime}, v^{\prime \prime}$, such that $x v=\alpha v^{\prime}, y v=v^{\prime}, z v=v^{\prime \prime}$, and such that $v^{\prime}$ and $v^{\prime \prime}$ are annihilated by $x, y, z$. That is,

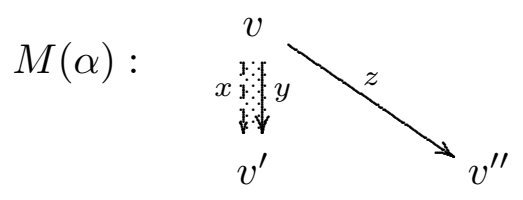


The modules $M(\alpha)$ with $\alpha \in k$ are pairwise non-isomorphic indecomposable $\Lambda$-modules.

For $\alpha \in k$, we define $m_{\alpha}=x-\alpha y \in \Lambda$. In order to provide a proof of Theorem 1.5, we now collect some general results for the modules $M(\alpha), \Lambda m_{\alpha}$, and the right ideals $m_{\alpha} \Lambda$ which are needed.

\subsection{The module $M(q)$.}

Lemma. The intersection of the kernels of all the homomorphisms $M(q) \rightarrow{ }_{\Lambda} \Lambda$ is $z M(q)=k v^{\prime \prime}$ and $M(q) / z M(q) \simeq \Lambda m_{1}$. In particular, $M(q)$ is not torsionless and $M(q)^{*}=\left(\Lambda m_{1}\right)^{*}$.

Proof. Let $f: M(q)=\Lambda v \rightarrow{ }_{\Lambda} \Lambda$ be a homomorphism. Let $f(v)=c_{1} x+c_{2} y+c_{3} z+$ $c_{4} y x+c_{5} z x$ with $c_{i} \in k$. By $q f\left(v^{\prime}\right)=f(x v)=x f(v)=-c_{2} q y x+c_{3} z x$ and $f\left(v^{\prime}\right)=f(y v)=$ $y f(v)=c_{1} y x$, we get $c_{2}=-c_{1}$ and $c_{3}=0$. Thus, $f(v)=c_{1}(x-y)+c_{4} y x+c_{5} z x$. It follows that $f\left(v^{\prime \prime}\right)=f(z v)=z f(v)=0$. This shows that $v^{\prime \prime}$ is contained in the kernel of any map $f: M(q)=\Lambda v \rightarrow{ }_{\Lambda} \Lambda$. On the other hand, the homomorphism $g: M(q)=\Lambda v \rightarrow \Lambda$ given by $g(v)=x-y=m_{1}$ has kernel $k v^{\prime \prime}$. This completes the proof of the first assertion.

The map $g$ provides a surjective map $p: M(q) \rightarrow \Lambda m_{1}$ and $p^{*}: M(q)^{*} \rightarrow\left(\Lambda m_{1}\right)^{*}$ is bijective, thus an isomorphism of right $\Lambda$-modules.

6.3. The modules $M(\alpha)$ with $\alpha \in k$. We consider now the modules $M(\alpha)$ in general, and relate them to the left ideals $\Lambda m_{\alpha}$, and to the right ideals $m_{\alpha} \Lambda$. Let us denote by $U_{\alpha}$ the twosided ideal generated by $m_{\alpha}$, it is 3 -dimensional with basis $m_{\alpha}, y x, z x$. Actually,

for any $\alpha \in k$, the right ideal $m_{\alpha} \Lambda$ is equal to $U_{\alpha}$ (but we prefer to write $U_{\alpha}$ instead of $m_{\alpha} \Lambda$ when we consider it as a left module). For $\alpha \neq 1$, the left ideal $\Lambda m_{\alpha}$ is equal to $U_{\alpha}$.

If $M$ is a module and $m \in M$, we denote by $r(m):{ }_{\Lambda} \Lambda \rightarrow M$ the right multiplication by $m$ (defined by $r(m)(\lambda)=\lambda m$. Similarly, if $N$ is a right $\Lambda$-module and $a \in N$, let $l(a): \Lambda_{\Lambda} \rightarrow N$ be the left multiplication by $a$.

We denote by $u_{\alpha}: \Lambda m_{\alpha} \rightarrow \Lambda$ and $u_{\alpha}^{\prime}: m_{\alpha} \Lambda \rightarrow \Lambda$ the canonical embeddings.

(1) The right ideal $m_{\alpha} \Lambda$ is 3-dimensional (and equal to $U_{\alpha}$ ), for all $\alpha \in k$.

(2) The left ideal $\Lambda m_{\alpha}$ is 3-dimensional (and equal to $U_{\alpha}$ ), for $\alpha \in k \backslash\{1\}$, whereas $\Lambda m_{1}$ is 2-dimensional.

(3) We have $M(\alpha) \simeq \Lambda / U_{\alpha}$ for all $\alpha \in k$.

Proof. The map $r(v): \Lambda \rightarrow M(\alpha)$ is surjective (thus a projective cover) and

$$
r(v)\left(m_{\alpha}\right)=m_{\alpha} v=(x-\alpha y) v=x v-\alpha y v=\alpha v^{\prime}-\alpha v^{\prime}=0 .
$$

Thus, $\Lambda m_{\alpha} \subseteq \operatorname{Ker}(r(v))$. Also, $z x \in \operatorname{Ker}(r(v))$, thus $\operatorname{Ker}(r(v))=U_{\alpha}$. This shows that $M(\alpha)$ is isomorphic to $\Lambda / U_{\alpha}$.

(4) For $\alpha \in k \backslash\{1\}$, we have $M(q \alpha) \simeq \Lambda m_{\alpha}$.

Proof. Consider the map $r\left(m_{\alpha}\right): \Lambda \rightarrow \Lambda m_{\alpha}$. Since $r\left(m_{\alpha}\right)\left(m_{q \alpha}\right)=m_{q \alpha} m_{\alpha}=0$, we see that $U_{q \alpha} \subseteq \operatorname{Ker}\left(r\left(m_{\alpha}\right)\right)$. For $\alpha \neq 1$, the module $\Lambda m_{\alpha}$ is 3-dimensional, therefore $r\left(m_{\alpha}\right)$ yields an isomorphism $\Lambda / U_{q \alpha} \rightarrow \Lambda m_{\alpha}$. Using (3) for $M(q \alpha)$, we see that $M(q \alpha) \simeq$ $\Lambda / U_{q \alpha} \simeq \Lambda m_{\alpha}$. 
(5) For any map $f: \Lambda m_{\alpha} \rightarrow \Lambda$, there is $\lambda \in \Lambda$ with $f=r(\lambda) u_{\alpha}$, for all $\alpha \in k$. Thus $u_{\alpha}$ is a left $\operatorname{add}(\Lambda)$-approximation.

Proof. Let $f: \Lambda m_{\alpha} \rightarrow \Lambda$ be any map. Let $f\left(m_{\alpha}\right)=c_{1} x+c_{2} y+c_{3} z+c_{4} y x+c_{5} z x$ with $c_{i} \in k$. Since $f\left(y m_{\alpha}\right)=f(y x)$ and $y f\left(m_{\alpha}\right)=c_{1} y x$, we see that $f(y x)=c_{1} y x$. Since $f\left(x m_{\alpha}\right)=f(-\alpha x y)=q \alpha f(y x)=q \alpha c_{1} y x$ and $x f\left(m_{\alpha}\right)=c_{2} x y+c_{3} z x=-q c_{2} y x+c_{3} z x$, we see that $q \alpha c_{1} y x=-q c_{2} y x+c_{3} z x$, therefore $c_{2}=-\alpha c_{1}$ and $c_{3}=0$. Thus, $f\left(m_{\alpha}\right)=$ $c_{1}(x-\alpha y)+c_{4} y x+c_{5} z x$ belongs to $U_{\alpha}=m_{\alpha} \Lambda$, say $f\left(m_{\alpha}\right)=m_{\alpha} \lambda$ with $\lambda \in \Lambda$. Therefore $f\left(m_{\alpha}\right)=m_{\alpha} \lambda=r(\lambda) u_{\alpha}\left(m_{\alpha}\right)$, but this means that $f=r(\lambda) u_{\alpha}$.

6.4. Lemma. Let $\alpha \in k \backslash\{1\}$. Then there is an $\mho$-sequence

$$
0 \rightarrow M(q \alpha) \rightarrow \Lambda \rightarrow M(\alpha) \rightarrow 0
$$

Proof. According to (3), $M(\alpha) \simeq \Lambda / U_{\alpha}$. Since $\alpha \neq 1$, we have $U_{\alpha}=\Lambda m_{\alpha}$ by (2). Thus, we have the following exact sequence

$$
0 \rightarrow \Lambda m_{\alpha} \stackrel{u_{\alpha}}{\rightarrow} \Lambda \rightarrow M(\alpha) \rightarrow 0
$$

According to (5) the embedding $u_{\alpha}: \Lambda m_{\alpha} \rightarrow \Lambda$ is a left add $(\Lambda)$-approximation. Thus, the sequence is an $\mho$-sequence. Finally, (4) shows that $\Lambda m_{\alpha} \simeq M(q \alpha)$.

Corollary 1. The module $M(0)$ is Gorenstein-projective with $\Omega$-period equal to 1.

Corollary 2. If $o(q)=\infty$, then the module $M(q)$ is semi-Gorenstein-projective.

Proof. We assume that $o(q)=\infty$. Then $q^{t} \neq 1$ for all $t \geq 1$. By 6.4, all the sequences

$$
0 \rightarrow M\left(q^{t+1}\right) \rightarrow \Lambda \rightarrow M\left(q^{t}\right) \rightarrow 0
$$

with $t \geq 1$ are $\mho$-sequences. They can be concatenated in order to obtain a minimal projective resolution of $M(q)$. This shows that $M(q)$ is semi-Gorenstein-projective.

6.5. The right $\Lambda$-modules $m_{\alpha} \Lambda$ and $M(\alpha)^{*}$. We have started in 6.3 to present essential properties of the modules $M(\alpha)$. We look now also at the modules $m_{\alpha} \Lambda$ and $M(\alpha)^{*}$. We continue the enumeration of the assertions as started in 6.3 .

(6) $\Omega\left(m_{q \alpha} \Lambda\right)=m_{\alpha} \Lambda$ for all $\alpha \in k$.

Proof. We consider the composition of the following right $\Lambda$-module maps

$$
\Lambda_{\Lambda} \stackrel{l\left(m_{\alpha}\right)}{\longrightarrow} \Lambda_{\Lambda} \stackrel{l\left(m_{q \alpha}\right)}{\longrightarrow} \Lambda_{\Lambda}
$$

Since $m_{q \alpha} m_{\alpha}=0$, the composition is zero. The image of $l\left(m_{\alpha}\right)$ is the right ideal $m_{\alpha} \Lambda$, the image of $l\left(m_{q \alpha}\right)$ is the right ideal $m_{q \alpha} \Lambda$. Both right ideals are 3 -dimensional, thus the sequence is exact. Thus $m_{\alpha} \Lambda=\operatorname{Ker}(p)$, for a surjective map $p: \Lambda_{\Lambda} \rightarrow m_{q \alpha} \Lambda$. Now $p$ is a projective cover, thus $\operatorname{Ker}(p)=\Omega\left(m_{q \alpha} \Lambda\right)$, and therefore $\Omega\left(m_{q \alpha} \Lambda\right) \simeq m_{\alpha} \Lambda$.

(7) $\left(\Lambda m_{\alpha}\right)^{*}=m_{\alpha} \Lambda$ for all $\alpha \in k$.

Proof. First, let us show that $\left(\Lambda m_{\alpha}\right)^{*}$ is 3-dimensional. On the one hand, besides $u_{\alpha}$, there are homomorphisms $\Lambda m_{\alpha} \rightarrow \Lambda$ with image $k y x$ and with image $k z x$, which shows that 
$\left(\Lambda m_{\alpha}\right)^{*}$ is at least 3-dimensional. According to (5), any homomorphism $\Lambda m_{\alpha} \rightarrow \Lambda$ maps into $\Lambda m_{\alpha} \Lambda=U_{\alpha}$. Since $U_{\alpha}$ is 3-dimensional, we have $\operatorname{dim} \operatorname{Hom}\left({ }_{\Lambda} \Lambda, U_{\alpha}\right)=3$, therefore $\operatorname{dim}\left(\Lambda m_{\alpha}\right)^{*}=\operatorname{dim} \operatorname{Hom}\left(\Lambda m_{\alpha}, \Lambda\right)=\operatorname{dim} \operatorname{Hom}\left(\Lambda m_{\alpha}, U_{\alpha}\right) \leq \operatorname{dim} \operatorname{Hom}\left(\Lambda \Lambda, U_{\alpha}\right)=3$.

Second, using again (5), we see that $\left(\Lambda m_{\alpha}\right)^{*}$ is, as a right $\Lambda$-module, generated by $u_{\alpha}$. Thus, there is a surjective homomorphism $\theta_{\alpha}: \Lambda_{\Lambda} \rightarrow\left(\Lambda m_{\alpha}\right)^{*}$ of right $\Lambda$-modules defined by $\theta_{\alpha}(1)=u_{\alpha}$. We have

$$
\left(\theta_{\alpha}\left(m_{q^{-1} \alpha}\right)\right)\left(m_{\alpha}\right)=\left(\theta_{\alpha}(1) m_{q^{-1} \alpha}\right)\left(m_{\alpha}\right)=\left(u_{\alpha} m_{q^{-1} \alpha}\right)\left(m_{\alpha}\right)=m_{\alpha} m_{q^{-1} \alpha}=0,
$$

therefore $\theta_{\alpha}\left(m_{q^{-1} \alpha}\right)=0$. It follows that $\theta_{\alpha}$ yields a surjective map $\Lambda_{\Lambda} / m_{q^{-1} \alpha} \Lambda \rightarrow\left(\Lambda m_{\alpha}\right)^{*}$. Actually, this map has to be an isomorphism, since $m_{q^{-1} \alpha} \Lambda$ is 3-dimensional. Therefore $\Lambda_{\Lambda} / m_{q^{-1} \alpha} \Lambda \simeq\left(\Lambda m_{\alpha}\right)^{*}$. By (6), $\Lambda_{\Lambda} / m_{q^{-1} \alpha} \Lambda \simeq m_{\alpha} \Lambda$. This completes the proof.

(8) $M(q \alpha)^{*}=m_{\alpha} \Lambda$ for all $\alpha \in k$.

Proof. For $\alpha \neq 1$, we have $M(q \alpha) \simeq \Lambda m_{\alpha}$ by (4), thus we use (7). For $\alpha=1$, we use 6.2 and then (7).

Let us stress that (7) and (8) show that $M(q)^{*}$ and $\left(\Lambda m_{1}\right)^{*}$ are isomorphic, namely isomorphic to $m_{1} \Lambda$, whereas $M(q)$ and $\Lambda m_{1}$ themselves are not isomorphic.

(9) Let $\alpha \in k \backslash\{1, q\}$. For any homomorphism $g: m_{\alpha} \Lambda \rightarrow \Lambda$ there is $\lambda \in \Lambda$ with $g=l(\lambda) u_{\alpha}^{\prime}$. Thus, $u_{\alpha}^{\prime}$ is a left $\operatorname{add}(\Lambda)$-approximation.

Proof: Let $g: m_{\alpha} \Lambda \rightarrow \Lambda_{\Lambda}$ be a homomorphism. We claim that $g\left(m_{\alpha}\right) \in \Lambda m_{\alpha}$. Let $g\left(m_{\alpha}\right)=c_{1} x+c_{2} y+c_{3} z+c_{4} y x+c_{5} z x$ with $c_{i} \in k$. Now, $g\left(m_{\alpha} x\right)=g(-\alpha y x)=-\alpha g(y x)$ and $g\left(m_{\alpha}\right) x=c_{2} x y+c_{3} z x$. Also, $g\left(m_{\alpha} y\right)=g(x y)=-q g(y x)$, and $g\left(m_{\alpha}\right) y=c_{1} x y+c_{3} z x=$ $-c_{1} q y x+c_{3} z x$, thus $g(y x)=-q^{-1} g\left(m_{\alpha} y\right)=-q^{-1}\left(-c_{1} q y x+c_{3} z x\right)=c_{1} y x-q^{-1} c_{3} z x$. It follows that $c_{2} y x+c_{3} z x=-\alpha g(y x)=-\alpha\left(c_{1} y x-q^{-1} c_{3} z x\right)=-\alpha c_{1} y x+\alpha q^{-1} c_{3} z x$. Therefore $c_{2}=-\alpha c_{1}$ and $c_{3}=\alpha q^{-1} c_{3}$. Since we assume that $\alpha \neq q$, it follows that $c_{3}=0$. Therefore $g\left(m_{\alpha}\right)=c_{1} x-\alpha c_{1} y+c_{3} z+c_{4} y x+c_{5} z x=c_{1}(x-\alpha y)+c_{4} y x+c_{5} z x$ belongs to $U_{\alpha}$. Since we also assume that $\alpha \neq 1$, we have $U_{\alpha}=\Lambda m_{\alpha}$. Thus $g\left(m_{\alpha}\right) \in \Lambda m_{\alpha}$.

As a consequence, there is $\lambda \in \Lambda$ with $g\left(m_{\alpha}\right)=\lambda m_{\alpha}$, therefore $g\left(m_{\alpha}\right)=\lambda m_{\alpha}=$ $l(\lambda) u_{\alpha}^{\prime}\left(m_{\alpha}\right)$. It follows that $g=l(\lambda) u_{\alpha}^{\prime}$.

6.6. Lemma. Let $\alpha \in k \backslash\{1, q\}$. Then there is an $\mho$-sequence of right $\Lambda$-modules

$$
0 \rightarrow m_{\alpha} \Lambda \stackrel{u_{\alpha}^{\prime}}{\rightarrow} \Lambda_{\Lambda} \rightarrow m_{q \alpha} \Lambda \rightarrow 0 .
$$

Proof. This is 6.5 (6) and (9).

6.7. Proof of Theorem 1.6. According to 6.5 (8), we have $M(q)^{*}=m_{1} \Lambda$. As we know from $6.2, M(q)$ is not torsionless.

We assume now that $o(q)=\infty$. The Corollary 2 in 6.4 shows that $M(q)$ is semiGorenstein-projective. Since $q^{-t} \neq 1$ for all $t \geq 1$, the sequences

$$
0 \rightarrow m_{q^{-t}} \Lambda \stackrel{u_{\alpha}^{\prime}}{\rightarrow} \Lambda_{\Lambda} \rightarrow m_{q^{-t+1}} \Lambda \rightarrow 0
$$

with $t \geq 1$ are $\mho$-sequences, by 6.6 . They can be concatenated in order to obtain a minimal projective resolution of $m_{1} \Lambda$ and show that $m_{1} \Lambda$ is semi-Gorenstein-projective. 
Finally, we want to show that $M(q)^{* *}=\Omega M(1)$. According to 6.3 (5), the map $u_{1}: \Lambda m_{1} \rightarrow \Lambda$ is a minimal left $\operatorname{add}(\Lambda)$-approximation, thus we may consider as in 2.4 (a) the following commutative diagram with exact rows:

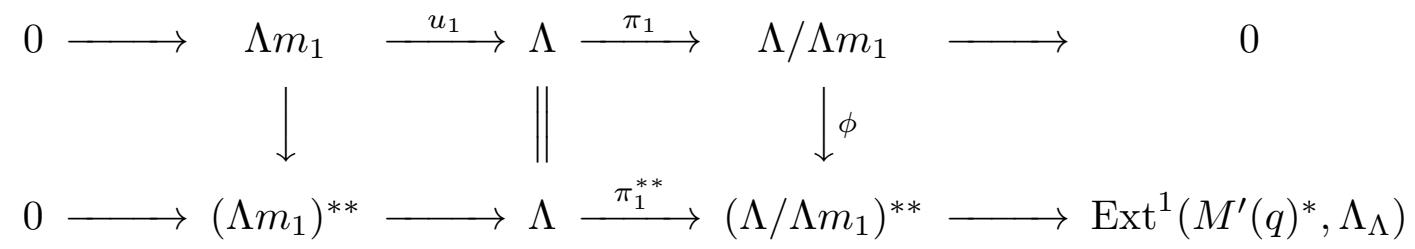

where $\phi=\phi_{\Lambda / \Lambda m_{1}}$. The submodule $z x\left(\Lambda / \Lambda m_{1}\right)$ belongs to the kernel of any map $\left.\Lambda / \Lambda m_{1}\right) \rightarrow$ $\Lambda$, and it is the kernel of the map $p: \Lambda / \Lambda m_{1} \rightarrow M(1)$ defined by $p(\overline{1})=v$. This shows that $z x\left(\Lambda / \Lambda m_{1}\right)$ is the kernel of $\phi$, thus the image of $\phi$ is just $M(1)$. But the image of $\phi$ coincides with the image of $\pi_{1}^{* *}$. In this way, we see that $\left(\Lambda m_{1}\right)^{* *}$ is the kernel of a projective cover of $M(1)$, thus equal to $\Omega M(1)$.

Of course, $\Omega M(1)$ is decomposable, namely isomorphic to $\Lambda m_{1} \oplus k z x$.

6.8. Proof of Addendum 1.6. We denote by $q^{\mathbb{Z}}$ the set of elements of $k$ which are of the form $q^{i}$ with $i \in \mathbb{Z}$. Assume that $\alpha \in k \backslash q^{\mathbb{Z}}$, thus $q^{t} \alpha \neq 1$ for all $t \in \mathbb{Z}$. According to 6.4 , all the sequences

$$
0 \rightarrow M\left(q^{t+1} \alpha\right) \rightarrow \Lambda \rightarrow M\left(q^{t} \alpha\right) \rightarrow 0
$$

with $t \in \mathbb{Z}$ are $\mho$-sequences. They can be concatenated in order to obtain a complete projective resolution for $M(\alpha)$, thus $M(\alpha)$ is Gorenstein-projective.

The following lemma shows that there are infinitely many elements $\alpha \in k \backslash q^{\mathbb{Z}}$.

Lemma. Assume that $k$ is an infinite field and $q \in k$. Then $k \backslash q^{\mathbb{Z}}$ is an infinite set.

We include a proof. The assertion is clear if $o(q)$ is finite. Thus, let $o(q)$ be infinite (in particular, $q \neq 0)$. Assume that the multiplicative group $k^{*}=k \backslash\{0\}$ is cyclic, say $k^{*}=w^{\mathbb{Z}}$. Then $o(w)=\infty$, and each element in $k^{*}$ different from 1 has infinite multiplicative order. Since $(-1)^{2}=1$, we see that $k$ is of characteristic 2 . Now $w+1 \neq 0$ shows that $w+1=w^{n}$ for some $n>1$, thus $w$ is algebraic over the prime field $\mathbb{Z}_{2}$. Thus $k=\mathbb{Z}_{2}(w)$ is a finite field, a contradiction. Since $k^{*}$ is not cyclic, there is $a \in k^{*} \backslash q^{\mathbb{Z}}$. Then $a \cdot q^{\mathbb{Z}}$ is an infinite subset of $k^{*} \backslash q^{\mathbb{Z}}$.

\section{Further details for $\Lambda=\Lambda(q)$.}

7.1. The $\mho$-components involving modules $M(\alpha)$. The only $\mho$-sequences which involve a module of the form $M(\alpha)$ with $\alpha \in k$ are those exhibited in 6.6.

Proof. We have to show that there is no $\mho$-sequence ending in $M(1)$ and no $\mho$-sequence starting in $M(q)$. Since $\Omega M(1)$ is decomposable, there is no $\mho$-sequence ending in $M(1)$. By 6.2 , the module $M(q)$ is not torsionless, thus no $\mho$-sequence starts in $M(q)$.

We now want to determine the $\mho$-type of the modules $M(\alpha)$. According to Corollary 1 in $6.4, M(0)$ is of $\mho$-type $\widetilde{\mathbb{A}}_{0}$. Thus, we now assume that $\alpha \neq 0$.

7.2. Let us assume that $o(q)=\infty$ (for the case that $o(q)$ is finite, see 7.6). There are three kinds of $\mho$-components which involve modules of the form $M(\alpha)$ with $\alpha \in k^{*}$. There 
is one component of the form $-\mathbb{N}$, it has $M(q)$ as its source, and there is one component of the form $\mathbb{N}$, it has $M(1)$ as its sink:

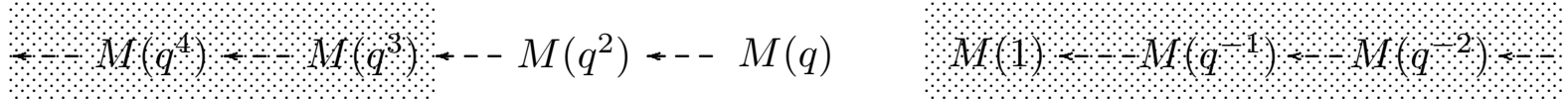

The remaining ones containing $M(\alpha)$ with $\alpha \neq 0$ and $\alpha \notin q^{\mathbb{Z}}$ are of the form $\mathbb{Z}$ :

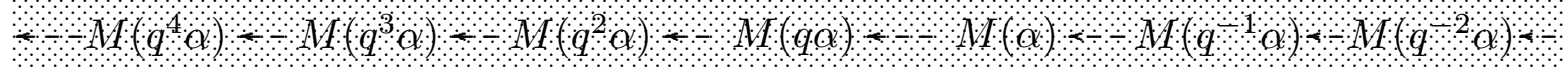

The positions of the reflexive modules are shaded.

According to Theorem 1.5, there are the following observations concerning the behavior of the modules $M(\alpha)$ with $\alpha \in k$.

- The module $M(\alpha)$ is Gorenstein-projective iff $\alpha \notin q^{\mathbb{Z}}$.

- The module $M(\alpha)$ is not Gorenstein-projective, but semi-Gorenstein-projective iff $\alpha=$ $q^{t}$ for some $t \geq 1$.

- The module $M(\alpha)$ is torsionless iff $\alpha \neq q$.

- The module $M(\alpha)$ is reflexive iff $\alpha \notin\left\{q, q^{2}\right\}$.

- The module $M(\alpha)$ is not Gorenstein-projective, but $\infty$-torsionfree iff $\alpha=q^{t}$ for some $t \leq 0$.

It seems worthwhile to know the canonical maps $\phi_{X}: X \rightarrow X^{* *}$ for the non-reflexive modules $X=M(q)$ and $X=M\left(q^{2}\right)$. For $M(q)$ we refer to 6.7: there it is shown that $M(q)^{* *}=\Omega M(1)$ and that the image of $\phi_{M(q)}$ is $\Lambda m_{1}$.

It remains to look at $X=M\left(q^{2}\right)$. The module $M\left(q^{2}\right)^{* *}$ is the submodule $\Lambda m_{q}+\Lambda z$ of $\Lambda$ and $\phi_{M\left(q^{2}\right)}$ is the inclusion map

$$
M\left(q^{2}\right)=\Lambda m_{q} \longrightarrow \Lambda m_{q}+\Lambda z=M\left(q^{2}\right)^{* *} .
$$

Proof. Since $M\left(q^{2}\right)$ is torsionless, the map $\phi_{M\left(q^{2}\right)}$ is injective. There is the following commutative diagram with exact rows:

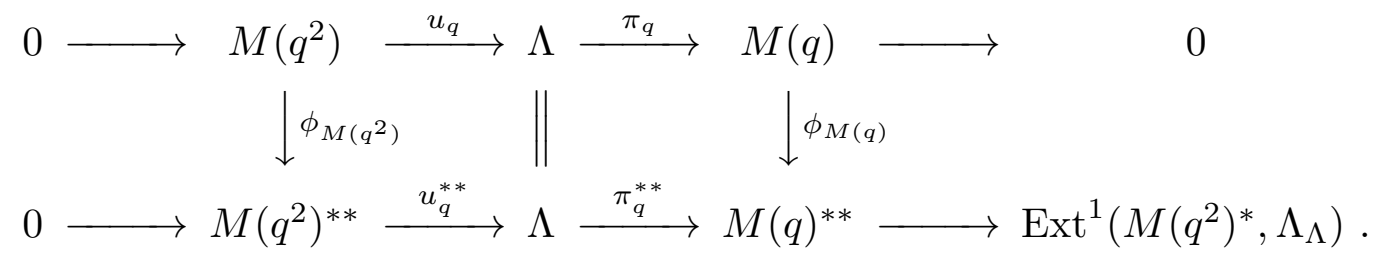

As we know already, the image of $\phi_{M(q)}$ and therefore of $\pi_{q}^{* *}$, is $\Lambda m_{1}$. Thus the kernel of $\pi_{q}^{* *}$ is the submodule $\Lambda m_{q}+\Lambda z$ of $\Lambda$. Therefore $M\left(q^{2}\right)^{* *}=\Lambda m_{q}+\Lambda z$ and $\phi_{M\left(q^{2}\right)}$ is the inclusion map $M\left(q^{2}\right)=\Lambda m_{q} \longrightarrow \Lambda m_{q}+\Lambda z=M\left(q^{2}\right)^{* *}$.

7.3. The $\mho$-components involving right $\Lambda$-modules $m_{\alpha} \Lambda$. The $\mho$-sequences which involve a right $\Lambda$-module of the form $m_{\alpha} \Lambda$ with $\alpha \in k$ are those exhibited in 6.6 as well as

$$
0 \rightarrow m_{q} \Lambda \stackrel{\left[\begin{array}{c}
u_{q} \\
h
\end{array}\right]}{\longrightarrow} \Lambda_{\Lambda} \oplus \Lambda_{\Lambda} \rightarrow \mho\left(m_{q} \Lambda\right) \rightarrow 0,
$$


and, for $q \neq 1$,

$$
0 \rightarrow m_{1} \Lambda \stackrel{\left[\begin{array}{l}
u_{1} \\
h^{\prime}
\end{array}\right]}{\longrightarrow} \Lambda_{\Lambda} \oplus \Lambda_{\Lambda} \rightarrow \mho\left(m_{1} \Lambda\right) \rightarrow 0 .
$$

Here, $h: m_{q} \Lambda \rightarrow \Lambda_{\Lambda}$ is defined by $h\left(m_{q}\right)=z$, whereas $h^{\prime}: m_{1} \Lambda \rightarrow \Lambda_{\Lambda}$ is defined by $h^{\prime}\left(m_{1}\right)=z x$.

Proof. It is easy to check that the map $\left[\begin{array}{c}u_{q} \\ h\end{array}\right]$ and, for $q \neq 1$, the map $\left[\begin{array}{l}u_{1} \\ h^{\prime}\end{array}\right]$ are minimal left add $\left(\Lambda_{\Lambda}\right)$-approximations. Clearly, the corresponding cokernels are not torsionless.

In addition, we have to show that there is no $\mho$-sequence ending in $m_{q^{2}} \Lambda$ or in $m_{q} \Lambda$. But this follows from the fact that the inclusion maps $u_{q}^{\prime}: m_{q} \Lambda=\Omega\left(m_{q^{2}} \Lambda\right) \rightarrow P\left(m_{q^{2}} \Lambda\right)$ and $u_{1}^{\prime}: m_{1} \Lambda=\Omega\left(m_{q} \Lambda\right) \rightarrow P\left(m_{q} \Lambda\right)$ are not add $\left(\Lambda_{\Lambda}\right)$-approximations.

Let $o(q)=\infty$ (the case that $o(q)<\infty$ will be discussed in 7.6). There are five kinds of $\mho$-components involving right $\Lambda$-modules of the form $m_{\alpha} \Lambda$ with $\alpha \in k$, namely a component of the form $\mathbb{N}$ with $m_{q^{2}} \Lambda$ as a sink, a component of the form $-\mathbb{N}$ with $\mho\left(m_{1} \Lambda\right)$ as a source, and a component of the form $\mathbb{A}_{2}$ with sink $m_{q} \Lambda$ and source $\mho\left(m_{q} \Lambda\right)$ :

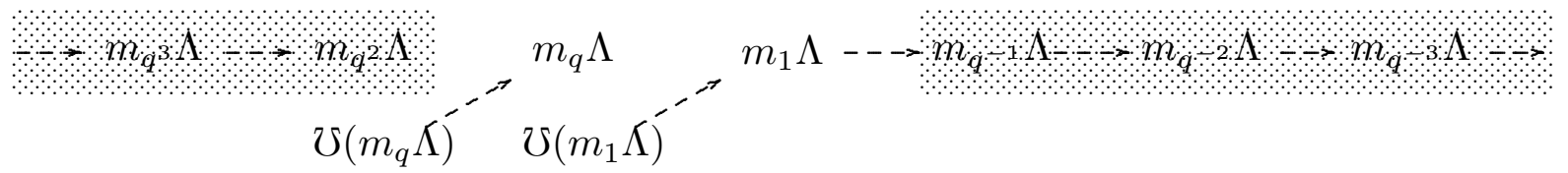

The $\mho$-components containing right $\Lambda$-modules $m_{\alpha} \Lambda$ with $\alpha \in k \backslash q^{\mathbb{Z}}$ are of the form $\mathbb{Z}$ :

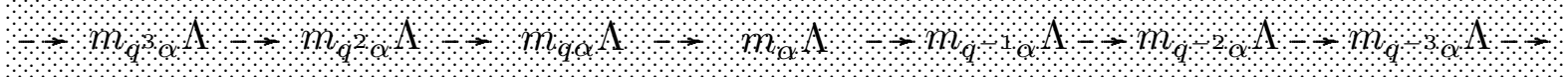

In addition, there is the $\mho$-component consisting of the single right $\Lambda$-modules $m_{0} \Lambda$, it is of the form $\widetilde{\mathbb{A}}_{0}$.

For the convenience of the reader, the pictures in 7.1 and 7.2 have been arranged so that the $A$-duality is respected. Thus, in 7.1, the arrows are drawn from right to left, in 7.2 from left to right. Also we recall from 6.3 (8) that the $A$-dual of $M(q \alpha)$ is $m_{\alpha} \Lambda$, therefore the position of $m_{\alpha} \Lambda$ in the pictures 7.2 is the same as the position of $M(q \alpha)$ in 7.1 .

7.4. We complete the description of the behavior of the modules $M(\alpha)$ started in 7.2 .

- The module $M(\alpha)$ is not Gorenstein-projective, but $M(\alpha)^{*}$ is semi-Gorenstein-projective, iff $\alpha=q^{t}$ for some $t \leq 1$.

- The module $M(\alpha)$ is not Gorenstein-projective, but $M(\alpha)^{*}$ is $\infty$-torsionfree, iff $\alpha=q^{t}$ for some $t \geq 3$.

Proof. According to 7.2, the module $M(\alpha)$ is Gorenstein-projective iff $\alpha \notin q^{\mathbb{Z}}$. Thus, we can assume that $\alpha=q^{t}$ for some $t \in \mathbb{Z}$. According to $6.3(8)$, the module $M\left(q^{t}\right)$ is isomorphic to $m_{q^{t-1}} \Lambda$. The display of the $\mho$-components shows that $m_{q^{t-1}} \Lambda$ is semiGorenstein-projective iff $t-1 \leq 0$, thus iff $t \leq 1$, see Theorem 1.5. Similarly, we see that $m_{q^{t-1}} \Lambda$ is $\infty$-torsionfree iff $t-1 \geq 2$, thus iff $t \geq 3$. 
7.5. We have mentioned in 1.7 that one may use the algebra $\Lambda=\Lambda(q)$ with $o(q)=\infty$ in order to exhibit examples of modules $M$ which satisfy precisely two of the three properties (G1), (G2) and (G3):

(1) $M=M(q)$ satisfies (G1), (G2), but not (G3).

(2) $M=M\left(q^{3}\right)$ satisfies (G1), (G3), but not (G2).

(3) $M=M(1)$ satisfies (G2), (G3), but not (G1).

Proof: For (1): this is the main assertion of Theorem 1.5. For (2): see 7.2 and 7.3. For (3): according to $7.2, M(1)$ is reflexive, but not Gorenstein-projective. According to $6.3(8)$, we have $M(1)^{*}=m_{q^{-1}} \Lambda$, and $m_{q^{-1}} \Lambda$ is semi-Gorenstein-projective, see 7.3 .

Let us look for similar examples for $\Lambda^{\mathrm{op}}$, thus, for right $\Lambda$-modules $N$.

(1*) There is no right $\Lambda$-module of the form $N=m_{\alpha} \Lambda$ satisfying (G1), (G2), but not (G3).

$\left(2^{*}\right)$ The right $\Lambda$-module $N=m_{q^{-2}} \Lambda$ satisfies (G1), (G3), but not (G2).

( $\left.3^{*}\right)$ The right $\Lambda$-module $N=m_{q^{2}} \Lambda$ satisfies (G2), (G3), but not (G1).

Proof: (2*) There starts an infinite $\mho$-path at $N=m_{q^{-2}} \Lambda$, thus $N$ satisfies (G1). There ends an $\mho$-path of length 2 at $N$, thus $N$ satisfies (G3). Of course, $N^{*}$ cannot be semi-Gorenstein-projective, since otherwise $N$ would be Gorenstein-projective.

$\left(3^{*}\right)$ Let $N=m_{q^{2}} \Lambda$. According to $6.5(8), N=M\left(q^{3}\right)^{*}$. As we know from $7.1, M\left(q^{3}\right)$ is reflexive, thus $N$ is reflexive and $N^{*}=M\left(q^{3}\right)^{* *}=M\left(q^{3}\right)$ is semi-Gorenstein-projective.

(1*) Assume that $N=m_{\alpha} \Lambda$ and $N^{*}$ are both semi-Gorenstein-projective. Since $N$ cannot be Gorenstein-projective, it is not reflexive. Thus $\alpha \in\{1, q\}$. Since $\left[m_{q} \Lambda\right]$ is the sink of an $\mho$-component, $m_{\alpha} \Lambda$ is not semi-Gorenstein-projective. Thus $\alpha=1$. But $\left(m_{1} \Lambda\right)^{*}=M(q)^{* *}=\Omega M(1)$, according to $6.5(8)$ and Theorem 1.5. As we have mentioned already in the proof $6.7, \Omega M(1) \simeq \Lambda m_{1} \oplus k$, where $k$ is the simple $\Lambda$-module. We claim that $k$ is not semi-Gorenstein-projective, thus $\Omega M(1)$ is not semi-Gorenstein-projective.

Lemma. Let $A$ be a local artin algebra which is not self-injective, and $S$ its simple $A$-module. Then $\operatorname{Ext}^{i}\left(S,{ }_{A} A\right) \neq 0$ for all $i \geq 1$.

Proof: Let $0 \rightarrow{ }_{A} A \rightarrow I_{0} \rightarrow I_{1} \rightarrow \cdots$ be a minimal injective coresolution. Since ${ }_{A} A$ is not injective, all the modules $I_{i}$ are non-zero. We have $\operatorname{Ext}^{i}\left(S,{ }_{A} A\right) \cong \operatorname{Hom}\left(S, I_{i}\right)$.

7.6. Let us look also at the case when $o(q)=n<\infty$.

Left modules $M(\alpha)$ with $\alpha \in k^{*}$ There are two kinds of $\mho$-components which involve modules of the form $M(\alpha)$ with $\alpha \in k^{*}$. There is one $\mho$-component of the form $\mathbb{A}_{n}$, it has $M(q)$ as its source, and $M(1)$ as its sink:

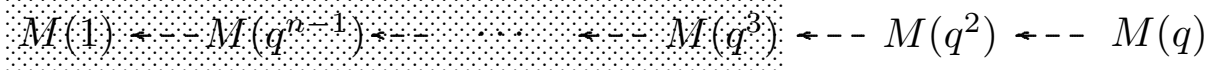

The remaining ones (containing the modules $M(\alpha)$ with $\alpha \in k^{*} \backslash q^{\mathbb{Z}}$ ) are directed cycles of cardinality $n$ :

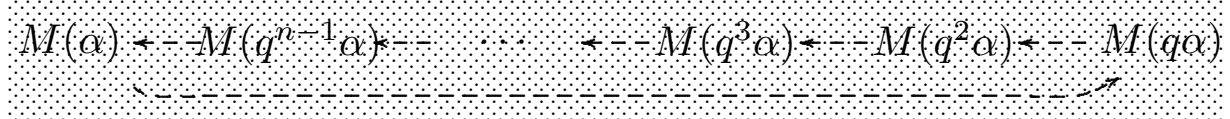

All modules in the cycles are reflexive. In the $\mho$-component of form $\mathbb{A}_{n}$, the modules $M(q)$ and $M\left(q^{2}\right)$ are not reflexive (they coincide for $o(q)=1$ ); for $o(q) \geq 3$, there are $n-2$ 
additional modules $M(1)=M\left(q^{n}\right), M\left(q^{n-2}\right), \cdots, M\left(q^{4}\right), M\left(q^{3}\right)$ in the $\mho$-component, and these modules are reflexive.

Right modules $m_{\alpha} \Lambda$ with $\alpha \in k^{*}$. There is always the $\mho$-component of form $\mathbb{A}_{2}$ with $\Omega\left(m_{q} \Lambda\right)$ as its source and $m_{q} \Lambda$ as its sink. In addition, for $n \geq 2$, there is an $\mho$ component of form $\mathbb{A}_{n}$ containing the modules $m_{q^{i}} \Lambda$ with $2 \leq i \leq n$ as well as $\Omega\left(m_{1} \Lambda\right)$; it has $\Omega\left(m_{1} \Lambda\right)$ as its source, and $m_{q^{2}} \Lambda$ as its sink:

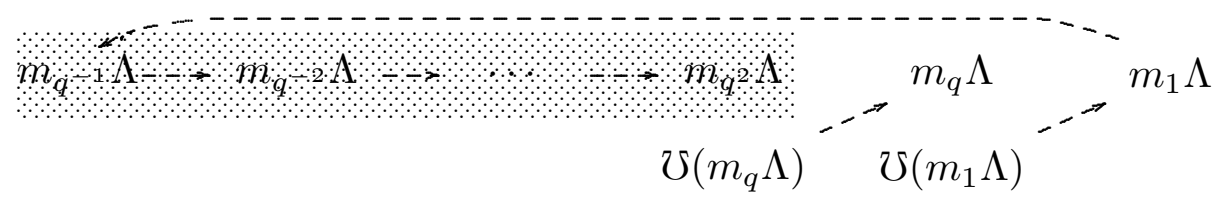

The remaining $\mho$-components (containing the right modules $m_{\alpha} \Lambda$ with $\alpha \in k^{*} \backslash q^{\mathbb{Z}}$ ) are directed cycles of cardinality $n$ :

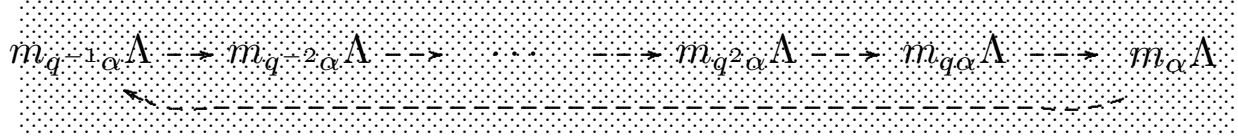

Again, the modules in the cycles are reflexive. In the $\mho$-components of form $\mathbb{A}_{n}$ and $\mathbb{A}_{2}$, the modules $m_{1} \Lambda$ and $\mho\left(m_{1} \Lambda\right)$, as well as $m_{q} \Lambda$ and $\mho\left(m_{q} \Lambda\right)$ are not reflexive; whereas (for $o(q) \geq 3)$ the modules $m_{q^{i}} \Lambda$ with $2 \leq i \leq n-1$ are reflexive.

Proof: First, let us look at left modules. According to 7.1, the $\mho$-sequences presented here are the only ones involving modules of the form $M(\alpha)$. Thus, $[M(q)]$ is a source in the $\mho$-quiver and $[M(1)]$ is a sink. This holds true also for $o(q)=1$ : here $q=1$ and $[M(1)]$ is both a sink and a source, thus a singleton $\mho$-component (without any arrow). Finally, for any $n$, the elements $1, q, \ldots, q^{n-1}$ are pairwise different, as are the elements $\alpha, q \alpha, \ldots, q^{n-1} \alpha$ for $\alpha \in k \backslash q^{\mathbb{Z}}$.

For dealing with the right modules, we refer to 7.3.

7.7. We have shown in 1.5 that any $\mho$-component is a linearly oriented quiver of type $\mathbb{A}_{n}$ (with $n \geq 1$ vertices), a directed cycle $\widetilde{\mathbb{A}}_{n}$ (with $n+1 \geq 1$ vertices), or of the form $-\mathbb{N}$, or $\mathbb{N}$, or $\mathbb{Z}$. Conversely, 7.2 and 7.6 show that all these cases arise for algebras of the form $\Lambda(q)$.

7.8. A forthcoming paper [RZ] will be devoted to a detailed study of all the 3 dimensional local $\Lambda$-modules for the algebra $\Lambda=\Lambda(q)$. If $q$ has infinite multiplicative order, we will encounter a whole family of 3-dimensional local modules which are semiGorenstein-projective, but not torsionless.

\section{Remarks.}

The first remarks draw the attention to the papers [JS] and $[\mathrm{CH}]$. In 8.1, we show that the $\Lambda(q)$-modules $M\left(q^{-s}\right)$ with $s \geq 0$ and $o(q)=\infty$ satisfy some further conditions which were discussed by Jorgensen and Şega. In 8.2 we show that the algebra $\Lambda(q)$ for $o(q)=\infty$ does not satisfy the so-called Auslander condition of Christensen and Holm.

In 8.3, we show that essential features of $\Lambda(q)$ are related to corresponding ones of its subalgebra $\Lambda^{\prime}(q)$, which is the quantum exterior algebra. 8.4 presents a two-fold covering 
of $\Lambda(q)$ which has properties similar to $\Lambda(q)$, but provides for $o(q)=\infty$ examples of semiGorenstein-projective modules $M$ which are not Gorenstein-projective, with the additional property that $\operatorname{End}(M)=k$.

8.1. The conditions $\left(\mathrm{TR}_{i}\right)$ of Jorgensen and Şega. As we have mentioned, Jorgensen and Şega have shown in [JS] that there exist semi-Gorenstein-projective modules which are not Gorenstein-projective. Actually, the main result of [JS] is a stronger assertion.

Following [JS], we say that an $R$-module $M$ satisfies the condition $\left(\mathrm{TR}_{i}\right)$ for some $i \geq 1$ provided $\operatorname{Ext}^{i}(M, R)=0$, and that $M$ satisfies the condition $\left(\mathrm{TR}_{i}\right)$ for some $i \leq-1$ provided $\operatorname{Ext}^{-i}\left(\operatorname{Tr} M, R_{R}\right)=0$. Note that $\left(\mathrm{TR}_{i}\right)$ is defined only for $i \neq 0$. Thus, $M$ is semi-Gorenstein-projective if and only if $M$ satisfies $\left(\mathrm{TR}_{i}\right)$ for all $i \geq 1$, and $M$ is $\infty$ torsionfree (i.e., $\operatorname{Tr} M$ is semi-Gorenstein-projective) if and only if $M$ satisfies ( $\left.\mathrm{TR}_{i}\right)$ for all $i \leq-1$. Note that $M$ satisfies $\left(\mathrm{TR}_{i}\right)$ if and only if $\operatorname{Tr}(M)$ satisfies $\left(\mathrm{TR}_{-i}\right)$. The main theorem of Jorgensen and Şega asserts that there exists a local artinian ring $R$ and a family $M_{s}$ of $R$-modules, with $M_{s}=\Omega M_{s+1}$ for $s \geq 1$, such that $M_{s}$ satisfies $\left(\mathrm{TR}_{i}\right)$ if and only if $i<s$.

Such a module $M_{s}$ satisfies the conditions (G2) and (G3), and satisfies in addition the condition that $\operatorname{Ext}^{i}\left(M_{s}, R\right)=0$ if and only if $1 \leq i \leq s-1$. Of course, this is a condition which is much stronger than the negation of (G1).

Let us show that our algebra $\Lambda(q)$ with $o(q)=\infty$ also provides such examples. Of course, in contrast to the algebra $R$ exhibited by Jorgensen and Şega, $\Lambda(q)$ is noncommutative. There is the following general result:

Proposition. Let $R$ be a local artinian algebra which is not self-injective, with simple $R$-module $S$.

If $M$ is an indecomposable $\infty$-torsionfree module such that $S$ is a proper direct summand of $\Omega M$, then $M$ satisfies $\left(\mathrm{TR}_{i}\right)$ if and only if $i<0$.

If $M$ is an indecomposable module such that $M$ satisfies $\left(\mathrm{TR}_{i}\right)$ if and only if $i<0$, then for every $s \geq 1$, the module $\mho^{s-1} M$ satisfies $\left(\mathrm{TR}_{i}\right)$ if and only if $i<s$.

Proof. First, let $M$ be indecomposable, $\infty$-torsionfree, with $\Omega M \cong S \oplus X$ for some non-zero module $X$. Since $M$ is $\infty$-torsionfree, $M$ satisfies $\left(\mathrm{TR}_{i}\right)$ for $i \leq-1$. Since $\Omega M$ is decomposable, we have $\operatorname{Ext}^{1}(M, R) \neq 0$, i.e., $M$ does not satisfy $\left(\mathrm{TR}_{1}\right)$. By Lemma 7.5, $\operatorname{Ext}^{i}(S, R) \neq 0$ for all $i \geq 1$. Thus, for $i \geq 2$ we have $\operatorname{Ext}^{i}(M, R) \cong \operatorname{Ext}^{i-1}(\Omega M, R) \cong$ $\operatorname{Ext}^{i-1}(S, R) \oplus \operatorname{Ext}^{i-1}(X, R) \neq 0$, which means that $M$ does not satisfy $\left(\mathrm{TR}_{i}\right)$.

Next, assume that $M$ is an indecomposable module such that $M$ satisfies $\left(\mathrm{TR}_{i}\right)$ if and only if $i \leq-1$. For $s \geq 1$ consider the module $M_{s}=\mho^{s-1} M$. For $i \leq-1, M_{s}$ satisfies satisfies $\left(\mathrm{TR}_{i}\right)$ : in fact, by Lemma $4.4, \operatorname{Ext}^{-i}\left(\operatorname{Tr}\left(M_{s}\right), R\right)=\operatorname{Ext}^{-i}\left(\operatorname{Tr}\left(\mho^{s-1} M\right), R\right) \cong$ $\operatorname{Ext}^{-i}\left(\operatorname{Tr}\left(\operatorname{Tr} \Omega^{s-1} \operatorname{Tr}(M)\right), R\right) \cong \operatorname{Ext}^{-i}\left(\Omega^{s-1} \operatorname{Tr}(M), R\right) \cong \operatorname{Ext}^{-i+s-1}(\operatorname{Tr}(M), R)=0$.

If $1 \leq i \leq s-1$, then $s-i \geq 1$ and $\operatorname{Ext}^{i}\left(M_{s}, R\right)=\operatorname{Ext}^{i}\left(\mho^{s-1} M, R\right) \cong \operatorname{Ext}^{1}\left(\mho^{s-i} M, R\right)=$ 0 , since $s-i-1 \geq 0$ and $\mho^{s-i-1} M$ is torsionless.

If $i \geq s$, then $i-s+1 \geq 1$ shows that $\operatorname{Ext}^{i}\left(M_{s}, R\right) \simeq \operatorname{Ext}^{i-s+1}(M, R) \neq 0$, i.e., $M_{s}$ does not satisfy $\left(\mathrm{TR}_{i}\right)$.

Application: Let $R=\Lambda=\Lambda(q)$ with $o(q)=\infty$. Then $M=M(1)$ is an indecomposable $\infty$-torsionfree module and $S$ is a proper direct summand of $\Omega M$, thus the Proposition above 
shows that for $s \geq 1, M_{s}=\mho^{s-1} M=M\left(q^{-(s-1)}\right)$ satisfies $\left(\mathrm{TR}_{i}\right)$ if and only if $i<s$.

8.2. The Auslander condition of Christensen and Holm. Christensen and Holm $[\mathrm{CH}]$ say that a left-noetherian ring $A$ satisfies the Auslander condition, provided that for every finitely generated left $A$-module $M$, there is an integer $b(M)$ with the following property: if $M^{\prime}$ is a finitely generated left $A$-module, then the vanishing $\operatorname{Ext}^{\gg 0}\left(M, M^{\prime}\right)=0$ implies that $\operatorname{Ext}^{>b(M)}\left(M, M^{\prime}\right)=0$. We are indebted to Christensen and Holm for having drawn our attention to Theorem $\mathrm{C}$ of $[\mathrm{CH}]$ which asserts: If $A$ is a finite-dimensional $k$-algebra $A$ satisfying the Auslander condition, then $A$ is left weakly Gorenstein (here, we have taken into account that a finite-dimensional $k$-algebra has a dualizing complex, see 3.4 in $[\mathrm{CH}])$. This shows that the algebra $\Lambda(q)$ with $o(q)=\infty$ does not satisfy the Auslander condition. Actually, this can be seen directly, using the following easy observation.

Proposition. Assume that $A$ is a finite-dimensional $k$-algebra which satisfies the Auslander condition. Let $N_{i}$ with $i \in \mathbb{Z}$ be finite-dimensional right $A$-modules with $\Omega N_{i}=$ $N_{i-1}$ for all $i$. If at least one of the modules $N_{i}$ is semi-Gorenstein-projective, then all the modules $N_{i}$ are semi-Gorenstein-projective, thus Gorenstein-projective.

Proof. Note that $A$ satisfies the Auslander condition if and only if for every finitedimensional right $A$-module $N$, there is an integer $c(N)$ such that for every finite-dimensional right $A$-module $N^{\prime}$, the vanishing $\operatorname{Ext}^{\gg 0}\left(N^{\prime}, N\right)=0$ implies that $\operatorname{Ext}^{>c(N)}\left(N^{\prime}, N\right)=0$ (here, $c(N)=b(D N)$, where $D=\operatorname{Hom}(,-, k)$ denotes the $k$-duality).

We assume that $N_{0}$ is semi-Gorenstein-projective, whereas $N_{1}$ is not semi-Gorensteinprojective. Then we must have $\operatorname{Ext}^{1}\left(N_{1}, A_{A}\right) \neq 0$. Since $N_{0}$ is semi-Gorenstein-projective, $\operatorname{Ext}^{t}\left(N_{0}, A_{A}\right)=0$ for all $t \geq 1$ and therefore $\operatorname{Ext}^{t+j}\left(N_{j}, A_{A}\right)=0$ for all $t \geq 1$ and $j \geq 0$. In particular, we have $\operatorname{Ext}^{\gg 0}\left(N_{j}, A_{A}\right)=0$ for all $j \geq 0$. Now we use the Auslander condition with $c=c\left(A_{A}\right)$. Since $\operatorname{Ext}^{\gg 0}\left(N_{c+1}, A_{A}\right)=0$, we have $\operatorname{Ext}^{c+1}\left(N_{c+1}, A_{A}\right)=0$. On the other hand, $\operatorname{Ext}^{c+1}\left(N_{c+1}, A_{A}\right) \simeq \operatorname{Ext}^{1}\left(N_{1}, A_{A}\right) \neq 0$. This is a contradiction.

For our algebra $\Lambda(q)$ with $o(q)=\infty$, let $N_{i}=m_{q^{i}} \Lambda$ with $i \in \mathbb{Z}$. According to 6.5 (6), we have $\Omega N_{i}=N_{i-1}$. As we know, the right module $N_{0}=m_{1} \Lambda=M(q)^{*}$ is semiGorenstein-projective, but not Gorenstein-projective, see Theorem 1.6. This shows that $\Lambda(q)$ with $o(q)=\infty$ does not satisfy the Auslander condition.

8.3. The quantum exterior algebra $\Lambda^{\prime}=\Lambda^{\prime}(q)$ in two variables (see, for example [S]). Let $\Lambda^{\prime}$ be the $k$-algebra generated by $x, y$ with the relations $x^{2}, y^{2}, x y+q y x$. It has a basis $1, x, y$, and $y x$. We may use the following picture as an illustration:

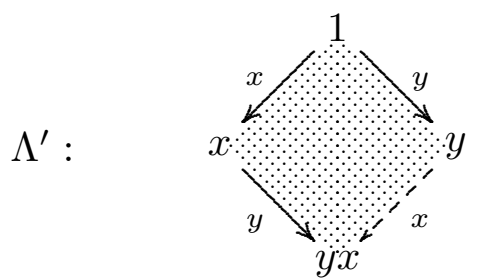

If we factor out the socle of $\Lambda^{\prime}$, we obtain the 3 -dimensional local algebra $\Lambda^{\prime \prime}$ with radical square zero (it is generated by $x, y$ with relations $x^{2}, y^{2}, x y, y x$ ).

Note that $\Lambda^{\prime}(q)$ is a subalgebra of $\Lambda(q)$, and that $\Lambda z \Lambda=\Lambda z=\operatorname{span}\{z, z x\}$. The composition $\Lambda^{\prime} \hookrightarrow \Lambda \rightarrow \Lambda / \Lambda z \Lambda$ of the canonical maps is an isomorphism of algebras. In 
this way, the $\Lambda^{\prime}$-modules may be considered as the $\Lambda$-modules which are annihilated by $z$. We should stress that the elements $m_{\alpha}=x-\alpha y$ (which play a decisive role in our investigation) belong to $\Lambda^{\prime}$.

For $\alpha \in k$, let $M^{\prime}(\alpha)$ be the $\Lambda^{\prime}$-module with basis $v, v^{\prime}$, such that $x v=\alpha v^{\prime}, y v=v^{\prime}$, and $x v^{\prime}=0=y v^{\prime}$. In addition, we define $M^{\prime}(\infty)$ as the $\Lambda^{\prime}$-module with basis $v, v^{\prime}$, such that $x v=v^{\prime}, y v=x v^{\prime}=y v^{\prime}=0$. Here are the corresponding illustrations:

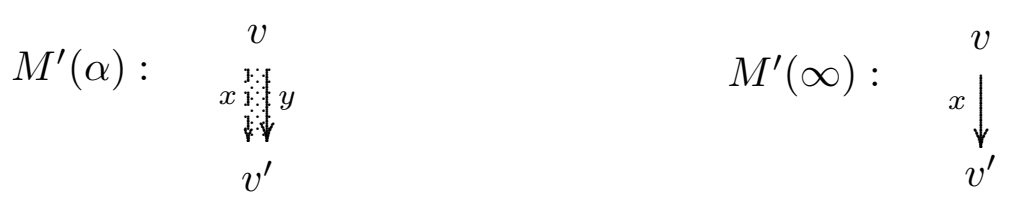

The modules $M^{\prime}(\alpha)$ with $\alpha \in k \cup\{\infty\}$ are pairwise non-isomorphic and indecomposable, and any two-dimensional indecomposable $\Lambda^{\prime}$-module is of this form. In particular, the left ideal $\Lambda^{\prime} m_{\alpha}$ is isomorphic to $M^{\prime}(q \alpha)$, for any $\alpha \in k \cup\{\infty\}$. The essential property of the modules $M^{\prime}(\alpha)$ is the following: $\Omega_{\Lambda^{\prime}} M^{\prime}(\alpha)=M^{\prime}(q \alpha)$. This follows from the fact that $m_{q \alpha} m_{\alpha}=0$ and it is this equality which has been used frequently in sections 6 and 7 .

For all $\alpha \in k, M(\alpha)$ considered as a $\Lambda^{\prime}$-module, is equal to $M^{\prime}(\alpha) \oplus k$, where $k$ is the simple $\Lambda^{\prime}$-module. Also, we should stress that $\operatorname{rad} \Lambda$ considered as a left $\Lambda^{\prime}$-module is the direct sum of $I$ and $M^{\prime}(\infty)$, where $I$ is the indecomposable injective $\Lambda^{\prime \prime}$-module.

8.4. A variation. Let $\widetilde{\Lambda}$ be the algebra defined by a quiver with two vertices, say labeled by 1 and 2 , with three arrows $1 \rightarrow 2$ labeled by $x, y, z$ and with three arrows $2 \rightarrow 1$, also labeled by $x, y, z$, satisfying the "same" relations as $\Lambda$ (of course, now we have 14 relations: seven concerning paths $1 \rightarrow 2 \rightarrow 1$ and seven concerning paths $2 \rightarrow 1 \rightarrow 2$ ). Whereas $\Lambda$ is a local algebra, the algebra $\widetilde{\Lambda}$ is a connected algebra with two simple modules $S(1)$ and $S(2)$.

For all the considerations in sections 6 and 7 , there are corresponding ones for $\widetilde{\Lambda}$, but always we have to take into account that now we deal with two simple modules $S(1)$ and $S(2)$ : Corresponding to the module $M(\alpha)$, there are two different modules, namely $M^{1}(\alpha)$ with top $S(1)$ and $M^{2}(\alpha)$ with top $S(2)$. The modules $M^{1}(\alpha)$ and $M^{2}(\alpha)$ have similar properties as $M(\alpha)$, in particular, $M^{1}(q)$ and $M^{2}(q)$ are semi-Gorenstein-projective and not Gorenstein-projective provided that $o(q)=\infty$. There is one decisive difference between the $\Lambda$-modules and the $\widetilde{\Lambda}$-modules: The endomorphism ring of $M^{1}(\alpha)$ and $M^{2}(\alpha)$ is equal to $k$, whereas the endomorphism ring of any $M(\alpha)$ is 3-dimensional.

\section{Questions.}

9.1. We have constructed a module which satisfies the conditions (G1), (G2), but not (G3). As we have mentioned already in the introduction, it is an open problem whether such a module does exist in case we deal with commutative rings.

9.2. One may ask whether or not the finiteness of $\operatorname{gp} A$ implies that $A$ is left weakly Gorenstein, There is a weaker question: is $A$ left weakly Gorenstein, in case all the Gorenstein-projective $A$-modules are projective?

9.3. Following Marczinzik [M1, question 1], one may ask whether a left weakly Gorenstein artin algebra is also right weakly Gorenstein, thus whether the existence of an $\mho$ component of the form $\mathbb{N}$ implies that also an $\mho$-component of the form $-\mathbb{N}$ exists. 
Note that if any right weakly Gorenstein algebra is left weakly Gorenstein, then the Gorenstein symmetry conjecture holds true. Namely, we claim: If inj. $\operatorname{dim} .{ }_{A} A \leq d$ and inj. $\operatorname{dim} . A_{A}>d$ (the Gorenstein symmetry conjecture asserts that this should not happen), then $A$ is right weakly Gorenstein, but not left weakly Gorenstein.

Proof. Let $Q$ be an injective cogenerator of $\bmod A$. We assume that inj. $\operatorname{dim} .{ }_{A} A$ is at most $d$. As we have seen in $4.9, A$ is right weakly Gorenstein and any module of the form $\Omega^{d} M$ is semi-Gorenstein projective. Now assume that $A$ is also left weakly Gorenstein. Then all the modules $\Omega^{d} M$ are Gorenstein-projective. In particular, $Q^{\prime}=\Omega^{d} Q$ is Gorenstein-projective. A well-known argument shows that if $Q^{\prime}$ is Gorenstein-projective, then $Q^{\prime}$ is even projective. [Namely, assume that $Q^{\prime}$ is Gorenstein-projective. Then there is a Gorenstein-projective module $Q^{\prime \prime}$ such that $Q^{\prime}=P^{\prime} \oplus \Omega^{d+1} Q^{\prime \prime}$ with $P^{\prime}$ projective. Now $\operatorname{Ext}^{1}\left(\Omega^{d} Q^{\prime \prime}, Q^{\prime}\right) \simeq \operatorname{Ext}^{d+1}\left(Q^{\prime \prime}, Q^{\prime}\right) \simeq \operatorname{Ext}^{1}\left(Q^{\prime \prime}, Q\right)=0$, here the first isomorphism is the usual index shift, whereas the second comes from the fact that $Q^{\prime \prime}$ is (semi-)Gorensteinprojective and $Q^{\prime}=\Omega^{d} Q$ (for a semi-Gorenstein-projective module $N$, and any module $Z$, we have $\operatorname{Ext}^{i+1}(N, \Omega Z) \simeq \operatorname{Ext}^{i}(N, Z)$ for all $\left.i \geq 1\right)$. But $\operatorname{Ext}^{1}\left(\Omega^{d} Q^{\prime \prime}, P^{\prime} \oplus \Omega^{d+1} Q^{\prime \prime}\right)=0$ implies that $\operatorname{Ext}^{1}\left(\Omega^{d} Q^{\prime \prime}, \Omega^{d+1} Q^{\prime \prime}\right)=0$, thus the canonical exact sequence $0 \rightarrow \Omega^{d+1} Q^{\prime \prime} \rightarrow$ $P\left(\Omega^{d} Q^{\prime \prime}\right) \rightarrow \Omega^{d} Q^{\prime \prime} \rightarrow 0$ splits and $\Omega^{d+1} Q^{\prime \prime}$ has to be projective (even zero). It follows that $Q^{\prime}=P^{\prime} \oplus \Omega^{d+1} Q^{\prime \prime}$ is projective.] Since $Q^{\prime}$ is projective, the projective dimension of $Q$ is at most $d$. Using duality, we see that inj. $\operatorname{dim} . A_{A} \leq d$.

9.4. Assume that there exists a non-reflexive $A$-module $M$ such that both $M$ and $M^{*}$ are semi-Gorenstein-projective. Is then the same true for $A^{\text {op? }}$ Even for $A=\Lambda(q)$ with $o(q)=\infty$, we do not know the answer. According to $7.5\left(1^{*}\right)$, a right $A$-module $N$ of the form $N=m_{\alpha} \Lambda(q)$ is reflexive, if both $N$ and $N^{*}$ are semi-Gorenstein-projective. But, there could exist some other right $A$-module $N$ satisfying (G1), (G2) and not (G3).

9.5. The Nunke condition. Does there exist a semi-Gorenstein-projective module $M \neq 0$ with $M^{*}=0$ ? Such a module would be an extreme example of a module satisfying (G1), (G2) and not (G3). Marczinzik has pointed out that this question concerns the Nunke condition $[\mathrm{H}]$ for $M$, which asserts that $\operatorname{Ext}^{i}(M, A) \neq 0$ for some $i \geq 0$, see $[\mathrm{J}]$. Colby and Fuller $[\mathrm{CF}]$ have conjectured that the Nunke condition should hold for any module $M$; they called this the strong Nakayama conjecture. The strong Nakayama conjecture obviously implies the generalized Nakayama conjecture which asserts that for any simple module $S$ there should exist some $i \geq 0$ such that $\operatorname{Ext}^{i}(S, A) \neq 0$. It is known that the Nunke condition is satisfied in case the finitistic dimension conjecture holds true.

Note that if $M$ is indecomposable and semi-Gorenstein-projective, then $M^{*}$ may be decomposable, as Theorem 1.5 shows: the $\Lambda(q)^{\text {op }}$ module $M(q)^{*}$ is indecomposable and semi-Gorenstein-projective, but $M(q)^{* *}$ is decomposable.

9.6. The conditions $\left(\mathrm{TR}_{i}\right)$. Following Jorgensen and Şega [JS], one may ask whether an $A$-module which satisfies $\left(\mathrm{TR}_{i}\right)$ for all but finitely many values of $i$, has to be Gorensteinprojective. In general, this is not the case, since there is the following proposition.

Proposition. If both $M$ and $M^{*}$ are semi-Gorenstein-projective, then $M$ satisfies the conditions $\left(\mathrm{TR}_{i}\right)$ for all $i \notin\{-1,-2\}$.

Proof. Let $M$ be semi-Gorenstein-projective. Then $M$ satisfies $\left(\mathrm{TR}_{i}\right)$ for $i \geq 1$. Since $\operatorname{Ext}^{1}(M, A)=0$ for $i=1,2$, Lemma 2.5 asserts that there is a projective module $Y$ such 
that $M^{*} \simeq \Omega^{2} \operatorname{Tr} M \oplus Y$. Assume now that also $M^{*}$ is semi-Gorenstein-projective. Then for $i \geq 1$, we have $\operatorname{Ext}^{i+2}\left(\operatorname{Tr} M, A_{A}\right)=\operatorname{Ext}^{i}\left(\Omega^{2} \operatorname{Tr} M, A_{A}\right)=\operatorname{Ext}^{i}\left(M^{*}, A_{A}\right)=0$, thus $M$ satisfies also $\left(\mathrm{TR}_{i}\right)$ for $i \leq-3$.

Thus, our paper shows that there are (non-commutative) artinian rings with modules $M$ which satisfy $\left(\mathrm{TR}_{i}\right)$ for all $i \notin\{-1,-2\}$ and which are not Gorenstein-projective. For commutative artinian rings (and this was the setting considered by Jorgensen and Şega) the question is open.

\section{References.}

[A1] M. Auslander, Anneaux de Gorenstein et torsion en algébre commutative, Sèminaire d'algébre commutative dirigé par P. Samuel (1966-1967), tome 1, notes by: M. Mangeney, C. Peskine, L. Szpiro, Secrétariat Mathématique, Paris, 2-69(1967).

[A2] M. Auslander, Coherent functors. In: Proc. the Conf. on Categorical Algebra. La Jolla 1965. Springer, 189-231.

[AB] M. Auslander, M. Bridger, Stable module theory, Mem. Amer. Math. Soc. 94., Amer. Math. Soc., Providence, R.I., 1969.

[AR1] M. Auslander, I. Reiten, Applications of contravariantly finite subcategories, Adv. Math. 86(1991), 111-152.

[AR2] M. Auslander, I. Reiten, Syzygy modules for Noetherian rings, J. Algebra 183 (1996), $167-185$.

[ARS] M. Auslander, I. Reiten, S. O. Smalø, Representation Theory of Artin Algebras, Cambridge Studies in Advanced Math. 36. Cambridge University Press, 1995.

[AM] L. L. Avramov, A. Martsinkovsky, Absolute, relative, and Tate cohomology of modules of finite Gorenstein dimension, Proc. London Math. Soc. 85(3)(2002), 393-440.

[B1] A. Beligiannis, The homological theory of contravariantly finite subcategories: auslanderbuchweitz contexts, gorenstein categories and (co-)stabilization, Comm. Algebra 28(10) (2000), 4547-4596.

[B2] A. Beligiannis, Cohen-Macaulay modules, (co)torsion pairs and virtually Gorenstein algebras, J. Algebra 288(1)(2005), 137-211.

[B3] A. Beligiannis, On algebras of finite Cohen-Macaulay type, Adv. Math. 226(2)(2011), 1973-2019.

[Br] M. Bridger, The $\operatorname{Ext}_{R}^{i}(M, R)$ and other invariants of $M$, Brandeis University, Mathematics. Ph.D. (1967).

[Buch] R.-O. Buchweitz, Maximal Cohen-Macaulay modules and Tate cohomology over Gorenstein rings, Unpublished manuscript, Hamburg (1987), 155pp.

[Che] X. W. Chen, Algebras with radical square zero are either self-injective or CM-free, Proc. Amer. Math. Soc. 140(1)(2012), 93-98.

[Chr] L. W. Christensen. Gorenstein Dimensions, Lecture Notes in Math. 1747, SpringerVerlag, 2000.

[CH] L. W. Christensen, H. Holm, Algebras that satisfy Auslander's condition on vanishing of cohomology, Math. Z. 265(2010), 21-40.

[CF] R. R. Colby, K. R. Fuller, A note on the Nakayama conjecture, Tsukuba J. Math. 14(1990), 343-352.

[EJ1] E. E. Enochs, O. M. G. Jenda, Gorenstein injective and projective modules, Math. Z. $220(4)(1995), 611-633$. 
[EJ2] E. E. Enochs, O. M. G. Jenda, Relative homological algebra, De Gruyter Exp. Math. 30. Walter De Gruyter Co., 2000.

$[\mathrm{H}]$ D. Happel, Homological conjectures in representation theory of finite-dimensional algebras, Unpublished. See: https://www.math.uni-bielefeld.de/ sek/dim2/happel2.pdf (retrieved Aug 6, 2018).

[HH] C. Huang, Z. Y. Huang, Torsionfree dimension of modules and self-injective dimension of rings, Osaka J. Math. 49(2012), 21-35.

[J] J. P. Jans, Duality in Noetherian rings, Proc. Amer. Math. Soc. 12(1961), 829-835.

[JS] D. A. Jorgensen, L. M. Şega, Independence of the total reflexivity conditions for modules, Algebras and Representation Theory 9(2)(2006), 217-226.

[K] B. Keller, Chain complexes and stable categories, Manuscripta Math. 67(1990), 379417.

[L] T. S. Lam, Lectures on Modules and Rings, Springer, 1999.

[M1] R. Marczinzik, Gendo-symmetric algebras, dominant dimensions and Gorenstein homological algebra, arXiv:1608.04212.

[M2] R. Marczinzik, On stable modules that are not Gorenstein projective, arXiv:1709.01132v3.

[R] C. M. Ringel, On the representation dimension of artin algebras, Bull. the Institute of Math., Academia Sinica, Vol. 7(1)(2012), 33-70.

[RX] C. M. Ringel, B. L. Xiong, Finite dimensional algebras with Gorenstein-projective nodes. In preparation.

[RZ] C. M. Ringel, P. Zhang, Gorenstein-projective and semi-Gorenstein-projective modules II, arXiv:1905.04048.

[S] S. O. Smalø, Local limitations of the Ext functor do not exist, Bull. London Math. Soc. 38(2006), 97-98.

[Y] Y. Yoshino, A functorial approach to modules of $G$-dimension zero, Illinois J. Math. $49(3)(2005), 345-367$.

[ZX] P. Zhang, B. L. Xiong. Separated monic representations II: Frobenius subcategories and RSS equivalences, Trans. Amer. Math. Soc. 372(2)(2019), 981-1021.

C. M. Ringel

Fakultät für Mathematik, Universität Bielefeld

POBox 100131, D-33501 Bielefeld, Germany

ringel@math.uni-bielefeld.de

P. Zhang

School of Mathematical Sciences, Shanghai Jiao Tong University

Shanghai 200240, P. R. China.

pzhang@sjtu.edu.cn 